\title{
DECIDABLE DISCRIMINATOR VARIETIES FROM UNARY CLASSES
}

\author{
ROSS WILLARD
}

\begin{abstract}
Let $\mathscr{K}$ be a class of (universal) algebras of fixed type. $\mathscr{K}^{t}$ denotes the class obtained by augmenting each member of $\mathscr{K}$ by the ternary discriminator function $(t(x, y, z)=x$ if $x \neq y, t(x, x, z)=z)$, while $\mathrm{V}\left(\mathscr{K}^{t}\right)$ is the closure of $\mathscr{K}^{t}$ under the formation of subalgebras, homomorphic images, and arbitrary Cartesian products. For example, the class of Boolean algebras is definitionally equivalent to $\mathrm{V}\left(\mathscr{K}^{t}\right)$ where $\mathscr{K}$ consists of a two-element algebra whose only operations are the two constants. Any equationally defined class (that is, variety) of algebras which is equivalent to some $\mathrm{V}\left(\mathscr{K}^{t}\right)$ is known as a discriminator variety.

Building on recent work of S. Burris, R. McKenzie, and M. Valeriote, we characterize those locally finite universal classes $\mathscr{K}$ of unary algebras of finite type for which the first-order theory of $\mathrm{V}\left(\mathscr{K}^{t}\right)$ is decidable.
\end{abstract}

\section{INTRODUCTION}

The attempt to determine which classes of models of finite type have a decidable first-order theory has a long and rich history. Recently R. McKenzie and $M$. Valeriote [15] have made great progress in the study of equationally defined classes of algebras (i.e., varieties) which are locally finite, by showing that such a variety is decidable iff it is the varietal product of three decidable locally finite varieties of very special kinds: strongly abelian, affine, and discriminator. Through the work of Valeriote $[19,15]$ it is completely understood which locally finite strongly abelian varieties are decidable. Though the same is not true of affine or discriminator varieties, it is known [3] that every locally finite affine variety is equivalent (from many points of view, including that of decidability) to the variety ${ }_{R} \mathscr{M}$ of all unitary left $R$-modules for some canonically defined finite ring $R$ with unit. Thus our understanding of decidable locally finite varieties will be complete if we can solve the following two problems (cf. [4, Chapter 14]).

Problem 1. For which finite rings $R$ with unit is ${ }_{R} \mathscr{M}$ decidable?

Problem 2. Which locally finite discriminator varieties of finite type are decidable?

This paper addresses the second problem.

Received by the editors October 9, 1990 and, in revised form, December 13, 1990.

1980 Mathematics Subject Classification (1985 Revision). Primary 03B25; Secondary 03B10, $03 \mathrm{D} 35$. 
For basic universal algebra the reader is referred to [5, 14]. An algebra is locally finite if each of its finitely generated subalgebras is finite; a class is locally finite if every algebra in it is locally finite. The ternary discriminator on the set $A$ is the function $t_{A}: A^{3} \rightarrow A$ given by

$$
t_{A}(a, b, c)= \begin{cases}c & \text { if } a=b, \\ a & \text { otherwise }\end{cases}
$$

A discriminator variety is a variety $\mathscr{V}$ for which there is a term $t(x, y, z)$ in the language of $\mathscr{V}$ which defines the ternary discriminator on the universe of every nontrivial subdirectly irreducible member of $\mathscr{V}$. An example of a locally finite discriminator variety is the variety of Boolean algebras. The only nontrivial subdirectly irreducible Boolean algebra (up to isomorphism) is the one whose universe is $\{0,1\}$, and every operation on $\{0,1\}$ is definable by a Boolean term.

There is a canonical way to generate discriminator varieties. Let $\mathscr{K}$ be a class of algebras of type $\mathrm{L}$, let $t$ be a ternary operation symbol not occurring in $\mathrm{L}$, and let $\mathrm{L}(t)$ denote the type $\mathrm{L} \cup\{t\}$. If $\mathbf{A} \in \mathscr{K}$ then $\mathbf{A}^{t}$ denotes the $\mathrm{L}(t)$-algebra $\left\langle\mathbf{A}, t_{A}\right\rangle$, and $\mathscr{K}^{t}$ denotes $\left\{\mathbf{A}^{t}: \mathbf{A} \in \mathscr{K}\right\}$. Then the variety $\mathrm{V}\left(\mathscr{K}^{t}\right)$ generated by $\mathscr{K}^{t}$ is a discriminator variety. Conversely, every discriminator variety is definitionally equivalent to one of the form $\mathrm{V}\left(\mathscr{K}^{t}\right)$ where $\mathscr{K}$ is an (essentially uniquely determined) universal class. Thus to solve Problem 2 it is enough to determine which locally finite universal classes $\mathscr{K}$ of algebras of finite type are such that $\mathrm{V}\left(\mathscr{K}^{t}\right)$ is decidable.

If $\mathscr{K}$ is a universal class of algebras, then the structure of the members of $\mathrm{V}\left(\mathscr{K}^{t}\right)$ can be described in terms of Boolean products.

Definition 1.1. A Boolean product of a nonempty indexed family $\left(\mathbf{A}_{x}\right)_{x \in X}$ of algebras is a subdirect product $\mathbf{A} \leq_{s d} \prod_{x \in X} \mathbf{A}_{x}$ of the family such that for some Boolean topology on $X$,

(1) For all $a, b \in A$, the set $\{x \in X: a(x)=b(x)\}$ is clopen;

(2) If $a, b \in A$ and $N$ is a clopen set of $X$, then $\left.a\right|_{N} \cup b_{X \backslash N} \in A$.

Condition (2) is known as the "patchwork property." We write $\mathbf{A} \leq_{b p}$ $\prod_{x \in X} \mathbf{A}_{x}$ if $\mathbf{A}$ is a Boolean product of $\left(\mathbf{A}_{x}\right)_{x \in X}$, and refer to the members of $\left(\mathbf{A}_{x}\right)_{x \in X}$ as the stalks of $\mathbf{A}$. For a class $\mathscr{K}, \Gamma^{a}(\mathscr{K})$ denotes the class of all Boolean products with stalks from $\mathscr{K}$. Usually when referring to a particular Boolean product of $\left(\mathbf{A}_{x}\right)_{x \in X}$ we shall assume that a particular Boolean topology on $X$ is specified.

If $\mathbf{A} \leq_{b p} \prod_{x \in X} \mathbf{A}_{x}$ then $A$ is a subuniverse of the $\mathrm{L}(t)$-algebra $\prod_{x \in X} \mathbf{A}_{x}^{t}$. We denote the corresponding subalgebra of $\prod_{x \in X} \mathbf{A}_{x}^{t}$ by $\mathbf{A}(t)$. Clearly $\mathbf{A}(t) \in$ $\mathrm{V}\left(\mathscr{K}^{t}\right)$. Conversely

Theorem 1.2 (Bulman-Fleming, Werner [1]). Suppose $\mathscr{K}$ is a universal class of algebras.

(i) If $\mathbf{B} \in \mathrm{V}\left(\mathscr{K}^{t}\right)$ and $|B|>1$, then $\mathbf{B} \cong \mathbf{A}(t)$ for some $\mathbf{A} \in \Gamma^{a}(\mathscr{K})$.

(ii) If moreover $\mathbf{B}$ is countable, then the Boolean product $\mathbf{A} \leq_{b p} \prod_{x \in X} \mathbf{A}_{x}$ referred to in (i) can be chosen so that the Boolean topology on $X$ has only countably many clopen sets.

Let $\mathscr{K}$ be a class of algebras of finite type $\mathrm{L} . \operatorname{Th}(\mathscr{K})$ denotes the set of all first-order L-sentences true in all members of $\mathscr{K} . \mathscr{K}$ is decidable if $\mathrm{Th}(\mathscr{K})$ 
is a recursive set; otherwise $\mathscr{K}$ is undecidable. $\mathscr{K}$ is hereditarily undecidable if every class $\mathscr{K}^{\prime}$ of L-algebras satisfying $\mathscr{K} \subseteq \mathscr{K}^{\prime}$ is undecidable. In this paper we establish the hereditary undecidability of a class $\mathscr{K}$ by semantically embedding into $\mathscr{K}$ a hereditarily undecidable class of graphs. The reader is referred to [3] for an explanation of the method. It suffices to note here that for any infinite cardinal $\kappa$ the class of all connected graphs with $\kappa$-many vertices is hereditarily undecidable.

Until 1988, only a few general results about the decidability of discriminator varieties were known. Tarski [18] showed in the 1940s that the variety of Boolean algebras is decidable. Ershov [9, 10] extended this result to several discriminator varieties not far removed from Boolean algebras. Then in the early 1970 s S. Comer [7, 8] developed some revolutionary sheaf-theoretic tools which allowed him to prove that for every $m \geq 2$ the (discriminator) variety of rings satisfying $x^{m}=x$ is decidable, and that every finitely generated variety of monadic algebras is decidable. H. Werner $[22,6]$ subsequently extended Comer's methods to show that every finitely generated discriminator variety of finite type is decidable. Apparently the first known example of a decidable but not finitely generated discriminator variety is the pure discriminator variety $\mathrm{V}\left(\mathrm{SETS}^{t}\right)$, shown to be decidable by McKenzie in 1976. Another example was given in [6].

For every $n \geq 1$ the class of cylindric algebras of dimension $n$ is a nonfinitely generated discriminator variety, which can easily be shown to be undecidable if $n>1$ [8]. In $1975 \mathrm{M}$. Rubin [17] showed that the same is true for dimension 1 (i.e., for monadic algebras). S. Burris subsequently generalized Rubin's result by showing [2] that if $\mathscr{V}$ is any nontrivial discriminator variety then $\mathrm{V}\left(\mathscr{V}^{t}\right)$ is undecidable.

Then in 1988 Burris, McKenzie, and Valeriote [4] developed some new and powerful tools to analyse certain locally finite but not finitely generated discriminator varieties. They were able to generalize McKenzie's result for $\mathrm{V}\left(\mathrm{SETS}^{t}\right)$ to prove that if $\mathscr{K}$ is a finitely axiomatizable locally finite universal class of finite type and $\mathscr{K}$ is locally homogeneous, then $\mathrm{V}\left(\mathscr{K}^{t}\right)$ is decidable; and that if $\mathscr{V}$ is a locally finite variety of finite unary type, then $\mathrm{V}\left(\mathscr{V}^{t}\right)$ is decidable iff $\mathscr{V}$ is locally homogeneous.

In this paper we present some stronger tools and a new notion of "almost local homogeneity" in order to classify those locally finite universal classes $\mathscr{K}$ of finite unary type for which $\mathrm{V}\left(\mathscr{K}^{t}\right)$ is decidable. This paper is a natural extension of the recent work of Burris, McKenzie, and Valeriote as well as of the earlier work of Comer and Werner.

We adopt the following conventions: $\mathscr{H}_{\text {fin }}$ denotes the class of all finite members of $\mathscr{K}$. An algebra is $n$-generated iff it is generated by a subset of cardinality at most $n$. A lower segment of a poset $\mathbf{P}$ is a subset $L$ of $P$ such that if $a \in L$ and $b \leq a$, then $b \in L$. If $\mathbf{A}$ is an algebra of type $\mathrm{L}$ and $a_{1}, \ldots, a_{n} \in A$, then the quantifier-free $n$-type of $\left\langle a_{1}, \ldots, a_{n}\right\rangle$ is the set of all quantifier-free L-formulas $\phi\left(x_{1}, \ldots, x_{n}\right)$ satisfying $\mathbf{A} \vDash \phi(\vec{a})$.

Burris and Valeriote first described their joint work with McKenzie at the University of Waterloo universal algebra seminar during the Spring 1988 and Winter 1989 terms, and provided us with an early draft of their manuscript [4]; we happily acknowledge our debt to them. We also thank Michael Albert, 
Burris, Keith Kearnes, McKenzie, and Valeriote for several helpful discussions and comments.

\section{A FefERman-VAught theOREM}

In this section we prove a Feferman-Vaught-style theorem for Boolean products in which (1) each stalk is homogeneous "modulo a designated finite subuniverse of bounded size," and (2) the designated finite subuniverses of the stalks are coordinated in a nice way. This theorem will be used in the following section.

Definition 2.1. (1) Suppose $\mathbf{D}$ is a locally finite algebra and $D_{0}$ is a subuniverse. $\mathbf{D}$ is homogeneous over $D_{0}$ if for all finite subalgebras $\mathbf{B}, \mathbf{B}^{\prime}$ of $\mathbf{D}$ satisfying $B \cap D_{0}=B^{\prime} \cap D_{0}$, every isomorphism $\sigma: \mathbf{B} \cong \mathbf{B}^{\prime}$ satisfying $\left.\sigma\right|_{B \cap D_{0}}=\mathrm{id}_{B \cap D_{0}}$ can be extended to an automorphism $\hat{\sigma}$ of $\mathbf{D}$ satisfying $\left.\hat{\sigma}\right|_{D_{0}}=\operatorname{id}_{D_{0}}$. D is homogeneous if it is homogeneous over $\mathrm{Sg}_{\mathrm{D}} \varnothing$.

(2) If $\mathbf{A}$ is a locally finite algebra and $A_{0}$ is a subuniverse, then $\mathbf{A}$ is locally homogeneous over $A_{0}$ if every finite subalgebra $\mathbf{D}$ of $\mathbf{A}$ is homogeneous over $D \cap A_{0} . \mathbf{A}$ is locally homogeneous if it is locally homogeneous over $\operatorname{Sg}_{\mathbf{A}} \varnothing$.

Here are two easy consequences of the previous definition.

Claim 2.2. Suppose $\mathbf{A}$ is locally finite, $A_{0}$ is a subuniverse, and $\mathbf{A}$ is locally homogeneous over $A_{0}$.

(i) If $\mathbf{A}^{\prime} \leq \mathbf{A}$, then $\mathbf{A}^{\prime}$ is locally homogeneous over $A^{\prime} \cap A_{0}$.

(ii) If $\mathbf{A}$ is countable, then $\mathbf{A}$ is homogeneous over $A_{0}$.

Proof. (i) is trivial. To prove (ii), suppose $\mathbf{B}, \mathbf{B}^{\prime}$ are finite subalgebras of $\mathbf{A}$ with $B \cap A_{0}=B^{\prime} \cap A_{0}$, and suppose $\sigma: \mathbf{B} \cong \mathbf{B}^{\prime}$ satisfies $\left.\sigma\right|_{B \cap A_{0}}=\mathrm{id}_{B \cap A_{0}}$. Let $D=\operatorname{Sg}_{\mathbf{A}}\left(B \cup B^{\prime}\right)$; then $\mathbf{D}$ is finite, so $\sigma$ can be extended to an automorphism $\hat{\sigma}$ of $\mathbf{D}$ satisfying $\left.\hat{\sigma}\right|_{D \cap A_{0}}=\operatorname{id}_{D \cap A_{0}}$. As $\mathbf{A}$ is the union of a chain of finite subalgebras containing $\mathbf{D}, \hat{\sigma}$ can be extended to an automorphism of $\mathbf{A}$ fixing $A_{0}$.

The next definition describes an artifice which serves only to simplify the presentation of the Feferman-Vaught theorem.

Definition 2.3. Suppose $\mathscr{K}$ is a locally finite universal class of similar algebras and $\mathbf{H}$ is a finite algebra of the same type.

(1) $\mathscr{K} / \mathbf{H}$ denotes the class of all $\mathbf{A} \in \mathscr{K}$ for which the set $A_{0}=A \cap H$ is a common subuniverse of $\mathbf{A}$ and $\mathbf{H}$ and, if nonempty, $\mathbf{A}_{0}$ inherits the same operations from $\mathbf{A}$ as it does from $\mathbf{H}$.

(2) Suppose $\mathbf{A}, \mathbf{B} \in \mathscr{K} / \mathbf{H}$. An $\mathbf{H}$-embedding from $\mathbf{A}$ to $\mathbf{B}$ is an embedding $\sigma: \mathbf{A} \hookrightarrow \mathbf{B}$ which satisfies $\sigma(a)=a$ for all $a \in A \cap H$ and $\sigma(a) \notin H$ for all $a \in A \backslash H$. In this case write $\sigma: \mathbf{A} \stackrel{\mathbf{H}}{\hookrightarrow} \mathbf{B}$. An $\mathbf{H}$-isomorphism is an isomorphism which is an $\mathbf{H}$-embedding in the above sense.

(3) If $\mathbf{A}, \mathbf{B} \in \mathscr{K} / \mathbf{H}$, write $\mathbf{A} \stackrel{\mathbf{H}}{\hookrightarrow} \mathbf{B}$ to mean there exists an $\mathbf{H}$-embedding $\sigma: \mathbf{A} \stackrel{\mathbf{H}}{\hookrightarrow} \mathbf{B}$, and $\mathbf{A} \stackrel{\mathbf{H}}{\cong} \mathbf{B}$ to mean there exists an $\mathbf{H}$-isomorphism from $\mathbf{A}$ to $\mathbf{B}$.

The following lemma contains the central property of $\mathbf{H}$-embeddings which we shall need. 
Lemma 2.4. Suppose $\mathscr{K}$ and $\mathbf{H}$ are as described in the previous definition and $\mathbf{A}, \mathbf{B}, \mathbf{C} \in \mathscr{K} / \mathbf{H}$ with $\mathbf{B}, \mathbf{C}$ finite and $\mathbf{B} \leq \mathbf{A}$. If $\mathbf{A}$ is locally homogeneous over $A \cap H, \alpha: \mathbf{B} \stackrel{\mathbf{H}}{\hookrightarrow} \mathbf{C}$, and $\mathbf{C} \stackrel{\mathbf{H}}{\hookrightarrow} \mathbf{A}$, then there exists $\beta: \mathbf{C} \stackrel{\mathbf{H}}{\hookrightarrow} \mathbf{A}$ satisfying $\beta \circ \alpha=\mathrm{id}_{B}$.

Proof. Pick $\gamma: \mathbf{C} \stackrel{\mathbf{H}}{\hookrightarrow} \mathbf{A}$ and let $B^{\prime}=\gamma \alpha(B)$ and $D=\operatorname{Sg}_{\mathbf{A}}(B \cup \gamma C)$. Note that $\gamma \alpha: \mathbf{B} \stackrel{\mathbf{H}}{\cong} \mathbf{B}^{\prime}$. As $\mathbf{D}$ is finite and $\mathbf{A}$ is locally homogeneous over $A \cap H, \gamma_{\alpha}$ extends to an $\mathbf{H}$-automorphism $\mu$ of $\mathbf{D}$. Let $\beta=\mu^{-1} \circ \gamma$.

Suppose now that $\mathscr{K}$ is a locally finite universal class of algebras of finite type for which $\mathrm{Th}_{\forall}(\mathscr{K})$ (the universal theory of $\mathscr{K}$ ) is decidable, and $\mathbf{H}$ is a finite algebra of the same type. Weispfennig observed in [21] that because the type of $\mathscr{K}$ is finite, there are only finitely many $n$-generated algebras in $\mathscr{K}$ (up to isomorphism) for each $n$. As $\mathrm{Th}_{\forall}(\mathscr{K})$ is decidable, there is an algorithm which, given $n \geq 0$, produces a finite set $\mathscr{K}_{n}$ of finite members of $\mathscr{K} / \mathbf{H}$ (explicitly constructed in some uniform manner) with the following properties:

1. Every $\mathbf{A} \in \mathscr{K}_{n}$ is $n$-generated;

2. For every $n$-generated $\mathbf{B} \in \mathscr{K} / \mathbf{H}$ there is an $\mathbf{A} \in \mathscr{K}_{n}$ such that $\mathbf{B} \stackrel{\mathbf{H}}{\cong} \mathbf{A}$;

3. For all $\mathbf{A}, \mathbf{B} \in \mathscr{K}_{n}$, if $\mathbf{A} \stackrel{\mathbf{H}}{\cong} \mathbf{B}$ then $\mathbf{A}=\mathbf{B}$;

4. $\mathscr{K}_{n} \subseteq \mathscr{K}_{n+1}$ for all $n \geq 0$.

In further discussions of $\mathscr{K} / \mathbf{H}$ in which the decidability of $\mathrm{Th}_{\forall}(\mathscr{K})$ is given, we shall assume that the above algorithm is fixed (hence the $\mathscr{K}_{n}$ 's are fixed) and shall let $\mathscr{K}_{\omega}=\bigcup_{n<\omega} \mathscr{K}_{n}$.

The recursiveness of $\mathscr{K}_{n}$ also implies the existence of an effective procedure which, given $n$, returns a finite set $T_{n}$ of terms in $x_{1}, \ldots, x_{n}$, with the property that for all $\mathbf{A} \in \mathscr{K}$ and all $\vec{a} \in A^{n}, \operatorname{Sg}_{\mathbf{A}}(\vec{a})=\left\{t^{\mathbf{A}}(\vec{a}): t \in T_{n}\right\}$. We shall refer to this family of sets $\left(T_{n}\right)_{n<\omega}$ in the proof of Theorem 2.7.

Now we turn to Boolean products.

Definition 2.5. Let $\mathscr{K}$ be a locally finite universal class of algebras of finite type, and $\mathbf{H}$ a finite algebra of the same type. $\Gamma_{\text {hom }}^{a}(\mathscr{K} / \mathbf{H})$ is the class of all Boolean products $\mathbf{A} \leq_{b p} \prod_{x \in X} \mathbf{A}_{x}$ in $\Gamma^{a}(\mathscr{K})$ which satisfy the following properties:

(1) $\mathbf{A}_{x} \in \mathscr{K} / \mathbf{H}$ for all $x \in X$;

(2) For all $a \in A$ and $h \in H$, the set $a^{-1}(h)$ is clopen;

(3) $\mathbf{A}_{x}$ is locally homogeneous over $A_{x} \cap H$ for all $x \in X$.

Definition 2.6. Let $\mathscr{K}$ and $\mathbf{H}$ be as above and such that $\operatorname{Th}_{\forall}(\mathscr{K})$ is decidable, and suppose $\mathbf{A} \leq_{b p} \prod_{x \in X} \mathbf{A}_{x}$ is in $\Gamma_{\text {hom }}^{a}(\mathscr{K} / \mathbf{H})$.

(1) For each $\mathbf{B} \in \mathscr{K}_{\omega}$ let $U_{\mathbf{B}}=\left\{x \in X: \mathbf{B} \stackrel{\mathbf{H}}{\leftrightarrow} \mathbf{A}_{x}\right\}$. Note that $U_{\mathbf{B}}$ is an open subset of $X$.

(2) $\vec{X}(\mathbf{A})=\left\langle X,\left(U_{\mathbf{B}}\right)_{\mathbf{B} \in \mathscr{K}_{\omega}}\right\rangle$.

(3) $\vec{X}(\mathbf{A})^{*}$ is the duai Boolean algebra with distinguished family of ideals indexed by $\mathscr{K}_{\omega}$.

Here is the promised Feferman-Vaught theorem.

Theorem 2.7. Suppose $\mathscr{K}$ is a locally finite universal class of algebras of finite type $\mathrm{L}$ such that $\mathrm{Th}_{\forall}(\mathscr{K})$ is decidable, and suppose $\mathbf{H}$ is a finite L-algebra. 
There is an effective procedure which, given an $\mathrm{L}(t)$ sentence $\phi$, produces a sentence $\Phi$ for Boolean algebras with distinguished ideals indexed by $\mathscr{K}_{\omega}$, such that for all $\mathbf{A} \in \Gamma_{\text {hom }}^{a}(\mathscr{K} / \mathbf{H})$,

$$
\mathbf{A}(t) \vDash \phi \quad \Leftrightarrow \quad \vec{X}(\mathbf{A})^{*} \vDash \Phi .
$$

Proof. The proof is a minor variation of the proof of Claim 3.3 in [4]. Suppose $\mathbf{A} \in \mathscr{K} / \mathbf{H}$ and $\vec{a} \in A^{n}$. By the quantifier-free $\mathbf{H}$ - $n$-type of $\vec{a}$ in $\mathbf{A}$ we mean some effective specification of the $n$-pointed algebra $\left\langle\operatorname{Sg}_{\mathbf{A}}(\vec{a}) ; \vec{a}\right\rangle$ up to H-isomorphism. (More precisely, if $\mathbf{B} \in \mathscr{K} / \mathbf{H}$ and $\vec{b} \in B^{n}$, then $\vec{a}$ and $\vec{b}$ have the same quantifier-free $\mathbf{H}$ - $n$-type in their respective algebras iff there exists $\alpha: \operatorname{Sg}_{\mathbf{A}}(\vec{a}) \stackrel{\mathbf{H}}{\hookrightarrow} \mathbf{B}$ satisfying $\alpha\left(a_{i}\right)=b_{i}$ for $i=1, \ldots, n$.) For the specification of the quantifier-free $\mathbf{H}-\boldsymbol{n}$-type of $\vec{a}$ in $\mathbf{A}$ we can take, for example, the ordered pair

$$
\left\langle\left\{\left(t_{1}, t_{2}\right) \in\left(T_{n}^{+}\right)^{2}: t_{1}^{\mathbf{A}}(\vec{a})=t_{2}^{\mathbf{A}}(\vec{a})\right\},\left\{(t, h) \in T_{n} \times H: t^{\mathbf{A}}(\vec{a})=h\right\}\right\rangle,
$$

where

$$
T_{n}^{+}=T_{n} \cup\left\{x_{1}, \ldots, x_{n}\right\} \cup\left\{f\left(t_{1}, \ldots, t_{k}\right): t_{1}, \ldots, t_{k} \in T_{n} \text { and } \quad f \in \mathrm{L} \text { is } k \text {-ary }\right\} .
$$

( $T_{n}$ was defined just before Definition 2.5.)

There are only finitely many quantifier-free $\mathbf{H}$ - $n$-types realized in $\mathscr{K} / \mathbf{H}$, and they are all realized in $\mathscr{K}_{n}$. Hence there is an effective procedure which, given $n$, enumerates all quantifier-free $\mathbf{H}-n$-types $p_{n, 1}, \ldots, p_{n, \lambda(n)}$ realized in $\mathscr{K} / \mathbf{H}$ and which, for each $(n, i)$, specifies a member $\mathbf{B}_{n, i} \in \mathscr{K}_{n}$ in which $p_{n, i}$ is realized.

The notation $p_{n+1, i} \vdash p_{n, j}$ means the obvious thing, i.e., if $\left(a_{1}, \ldots, a_{n+1}\right)$ in A has quantifier-free $\mathbf{H}-(n+1)$-type $p_{n+1, i}$, then $\left(a_{1}, \ldots, a_{n}\right)$ has type $p_{n, j}$. Likewise, if $\phi\left(x_{1}, \ldots, x_{n}\right)$ is a quantifier-free formula then $p_{n, i} \vdash \phi$ means A $\vDash \phi(\vec{a})$ whenever $\vec{a}$ has type $p_{n, i}$. Note that the predicates " $p_{n+1, i} \vdash p_{n, j}$ " and " $p_{n, i} \vdash \phi$ " are recursive.

Now suppose $\mathbf{A} \leq_{b p} \prod_{x \in X} \mathbf{A}_{x}$ is in $\Gamma_{\text {hom }}^{a}(\mathscr{K} / \mathbf{H})$ and $\vec{a} \in A^{n}$. By $\llbracket p_{n, i}(\vec{a}) \rrbracket$ we mean the set of those $x \in X$ for which the quantifier-free H- $n$-type of $\left\langle a_{1}(x), \ldots, a_{n}(x)\right\rangle$ in $\mathbf{A}_{x}$ is $p_{n, i}$. By condition (2) of Definition 2.5, $\llbracket p_{n, i}(\vec{a}) \rrbracket$ is clopen.

Claim 2.8. Suppose $\mathbf{A} \leq_{b p} \prod_{x \in X} \mathbf{A}_{x}$ is in $\Gamma_{\text {hom }}^{a}(\mathscr{K} / \mathbf{H})$ and $\vec{a} \in A^{n}$. Then for any clopen sets $N_{1}, \ldots, N_{\lambda(n+1)}$ of $X$,

$$
\left(N_{i}\right)_{i=1}^{\lambda(n+1)}=\left(\llbracket p_{n+1, i}(\vec{a}, b) \rrbracket\right)_{i=1}^{\lambda(n+1)} \text { for some } b \in A,
$$

iff

(1) $\bigcup_{i=1}^{\lambda(n+1)} N_{i}=X$, and $N_{i} \cap N_{j}=\varnothing$ whenever $i \neq j$;

(2) $N_{i} \subseteq \llbracket p_{n, j}(\vec{a}) \rrbracket$ for all $i, j$ such that $p_{n+1, i} \vdash p_{n, j}$; and

(3) $N_{i} \subseteq U_{\mathbf{B}_{n+1, i}}$ for all $i=1, \ldots, \lambda(n+1)$.

Proof. $(\Rightarrow)$ is easy to verify.

$(\Leftarrow)$. Suppose conditions (1)-(3) hold. By virtue of (1), the compactness of $X$, and the patchwork property of $A$, it will suffice to show that for each $i=1, \ldots, \lambda(n+1)$ and $x \in N_{i}$ there exists $b \in A_{x}$ such that the quantifier-free $\mathbf{H}-(n+1)$-type of $\left\langle a_{1}(x), \ldots, a_{n}(x), b\right\rangle$ is $p_{n+1, i}$. Let 
$B=\operatorname{Sg}_{\mathbf{A}_{x}}\left(a_{1}(x), \ldots, a_{n}(x)\right)$, and suppose the type of $\left\langle a_{1}(x), \ldots, a_{n}(x)\right\rangle$ is $p_{n, j}$. Let $k$ be the unique integer such that $p_{n+1, i} \vdash p_{n, k}$; then condition (2) and the fact that $N_{i} \cap \llbracket p_{n, j}(\vec{a}) \rrbracket \neq \varnothing$ imply $k=j$. Hence there exist $\alpha: \mathbf{B} \stackrel{\mathbf{H}}{\hookrightarrow} \mathbf{B}_{n+1, i}$ and $c \in B_{n+1, i}$ such that $\left\langle\alpha a_{1}(x), \ldots, \alpha a_{n}(x), c\right\rangle$ has type $p_{n+1, i}$.

By condition (3), $\mathbf{B}_{n+1, i} \stackrel{\mathbf{H}}{\hookrightarrow} \mathbf{A}_{x}$; thus Lemma 2.4 gives an $\mathbf{H}$-embedding $\beta: \mathbf{B}_{n+1, i} \stackrel{\mathbf{H}}{\hookrightarrow} \mathbf{A}_{x}$ satisfying $\beta \circ \alpha=\mathrm{id}_{B}$. As $\mathbf{H}$-embeddings preserve quantifierfree H-types, $\left\langle a_{1}(x), \ldots, a_{n}(x), \beta(c)\right\rangle$ has type $p_{n+1, i}$ in $\mathbf{A}_{x}$ as desired.

Now to prove the theorem, we shall give a recursive procedure which, given an $\mathrm{L}(t)$-formula $\phi\left(x_{1}, \ldots, x_{n}\right)$, produces a formula $\Phi\left(X_{1}, \ldots, X_{\lambda(n)}\right)$ in the firstorder language for Boolean algebras with a family $\left(I_{\mathbf{B}}\right)_{\mathbf{B} \in \mathscr{K}_{\omega}}$ of distinguished ideals ${ }^{1}$, such that for all $\mathbf{A} \in \Gamma_{\text {hom }}^{a}(\mathscr{K} / \mathbf{H})$ and all $\vec{a} \in A^{n}$,

$$
\mathbf{A}(t) \vDash \phi(\vec{a}) \quad \text { iff } \quad \vec{X}(\mathbf{A})^{*} \vDash \Phi\left(\llbracket p_{n, 1}(\vec{a}) \rrbracket, \ldots, \llbracket p_{n, \lambda(n)}(\vec{a}) \rrbracket\right) .
$$

The procedure is:

1. If $\phi\left(x_{1}, \ldots, x_{n}\right)$ is atomic, then $\Phi\left(X_{1}, \ldots, X_{\lambda(n)}\right)$ is $\left(1=\bigcup_{p_{n, i} \vdash \phi} X_{i}\right)$.

2. If $\phi$ is $\neg \theta$ and $\theta$ corresponds to $\Theta$, then $\Phi$ is $\neg \Theta$.

3. If $\phi$ is $\theta_{1} \& \theta_{2}$ and $\theta_{i}$ corresponds to $\Theta_{i}$ for $i=1,2$, then $\Phi$ is $\Theta_{1} \& \Theta_{2}$.

4. If $\phi$ is $\exists x_{n+1} \theta\left(x_{1}, \ldots, x_{n+1}\right)$ and $\theta$ corresponds to $\Theta\left(X_{1}, \ldots, X_{\lambda(n+1)}\right)$, then $\Phi\left(X_{1}, \ldots, X_{\lambda(n)}\right)$ is

$$
\begin{aligned}
\exists Y_{1} \cdots Y_{\lambda(n+1)} \quad & {\left[1=\bigcup_{i=1}^{\lambda(n+1)} Y_{i} \& \bigwedge_{i<j}\left(Y_{i} \cap Y_{j}=0\right) \&\right.} \\
& \left.\bigwedge_{p_{n+1, i}+p_{n, j}}\left(Y_{i} \subseteq X_{j}\right) \& \bigwedge_{i=1}^{\lambda(n+1)} I_{\mathbf{B}_{n+1, i}}\left(Y_{i}\right) \& \Theta\left(Y_{1}, \ldots, Y_{\lambda(n+1)}\right)\right] .
\end{aligned}
$$

That the procedure does what was claimed is proved by induction on the complexity of $\phi$, using compactness and the patchwork property when $\phi$ is atomic, and using Claim 2.8 when $\phi$ is of the form $\exists x_{n+1} \theta$.

\section{Decidability}

Definition 3.1. Suppose $\mathscr{K}_{0}$ is a finite set of finite algebras and $\mathbf{A}$ is an algebra of the same type. A maximal $\mathscr{K}_{0}$-subuniverse of $\mathbf{A}$ is a subuniverse $A_{0}$ such that (i) either $A_{0}=\varnothing$ or $\mathbf{A}_{0}$ is isomorphic to some member of $\mathscr{K}_{0}$, and (ii) $A_{0}$ is maximal (among subuniverses of $\mathbf{A}$ ordered by inclusion) with respect to property (i).

Definition 3.2. Suppose $\mathscr{K}$ is a locally finite universal class of algebras of finite type. We shall say that $\mathscr{K}$ is almost locally homogeneous ${ }^{2}$ if there is a finite set $\mathscr{K}_{0}$ of finite members of $\mathscr{K}$ satisfying.

(1) $\mathrm{S}\left(\mathscr{K}_{0}\right) \subseteq I\left(\mathscr{K}_{0}\right)$;

(2) (If the type of $\mathscr{K}$ contains constant symbols): Every 0-generated member of $\mathscr{K}$ is in $I\left(\mathscr{K}_{0}\right)$;

\footnotetext{
${ }^{1}$ Interpreted as unary relations.

${ }^{2}$ We thank the referee for suggesting this terminology.
} 
(3) If $\mathbf{A} \in \mathscr{K}$ and $A_{0}$ is a maximal $\mathscr{K}_{0}$-subuniverse of $\mathbf{A}$, then $\mathbf{A}$ is locally homogeneous over $A_{0}$.

If $\mathscr{K}$ is a locally finite universal class of finite type which is either locally homogeneous or of the form $\mathscr{K}=I\left(\mathscr{K}_{0}\right)$ for some finite set $\mathscr{K}_{0}$ of finite algebras, then $\mathscr{K}$ is almost locally homogeneous. Thus the next theorem is a common generalization of the decidability results of Burris, McKenzie, and Valeriote [4]; and of Werner [22, 6].

Theorem 3.3. Suppose $\mathscr{K}$ is a locally finite universal class of finite type. If $\mathrm{Th}_{\forall}(\mathscr{K})$ is decidable (for example, if $\mathscr{K}$ is finitely axiomatizable) and $\mathscr{K}$ is almost locally homogeneous, then $\mathrm{V}\left(\mathscr{K}^{t}\right)$ is decidable.

Remark 1. Suppose $\mathscr{K}$ is a finitely axiomatizable class of finite type. Then as is well known, $\operatorname{Th}(\mathscr{K})$, the full first-order theory of $\mathscr{K}$, and FinSat $(\mathscr{K})$, the set of first-order sentences true in some finite member of $\mathscr{K}$, are both recursively enumerable. If in addition $\mathscr{K}$ is universal and locally finite, then any universal sentence not belonging to $\mathrm{Th}(\mathscr{K})$ must be false in some finite member of $\mathscr{K}$, and hence its negation will belong to FinSat $(\mathscr{K})$. Thus $\operatorname{Th}_{\forall}(\mathscr{K})$ will be recursive, which explains the parenthetical remark in the statement of the theorem.

Remark 2. It is not hard to show that both the hypotheses and the conclusion of Theorem 3.3 are invariant under the transformation of $\mathscr{K}$ which replaces each constant by a constant unary operation. Therefore we shall prove the theorem under the additional hypothesis that the type of $\mathscr{K}$ contains no constant symbols. For the remainder of this section, let $\mathscr{K}$ be a fixed class satisfying the hypotheses of Theorem 3.3 and this additional hypothesis. We need the following two lemmas, whose proofs will be deferred to the end of the section.

Lemma 3.4. There exists a finite algebra $\mathbf{H}$ of the same type as $\mathscr{K}$, such that for every countable member $\mathbf{A}$ of $\Gamma^{a}(\mathscr{K})$ there exists $\mathbf{A}^{\prime} \in \Gamma_{\text {hom }}^{a}(\mathscr{K} / \mathbf{H})$ having the same underlying Boolean space as $\mathbf{A}$ and satisfying $\mathbf{A}(t) \cong \mathbf{A}^{\prime}(t)$.

Lemma 3.5. There is an effectively computable function from the collection of finite subsets of $\mathscr{K}_{\omega}$ to the set of natural numbers, written $\mathscr{F} \mapsto N_{\mathscr{F}}$, with the following property: For each $\mathscr{F}$, and for every $\mathbf{A} \leq_{b p} \prod_{x \in X} \mathbf{A}_{x}$ in $\Gamma_{\text {hom }}^{a}(\mathscr{K} / \mathbf{H})$ for which the Boolean algebra $X^{*}$ of clopen sets of $X$ is countable, there is a subalgebra $\mathbf{A}^{\prime} \leq \mathbf{A}$ such that

(i) $\mathbf{A}^{\prime} \in \Gamma_{\text {hom }}^{a}(\mathscr{K} / \mathbf{H})$;

(ii) $U_{\mathbf{B}}^{\prime}=U_{\mathbf{B}}$ for all $\mathbf{B} \in \mathscr{F}$;

(iii) $\mathbf{A}_{x}^{\prime}$ is $N_{\mathscr{F}}$-generated for all $x \in X$.

Proof of Theorem 3.3. Given an $\mathrm{L}(t)$-sentence $\phi$, we wish to decide whether $\mathrm{V}\left(\mathscr{K}^{t}\right) \vDash \phi$. First compute the Boolean sentence $\Phi$ as in Theorem 2.7; next let $\mathscr{F}$ be the set of indices of ideal symbols occurring in $\Phi$; then compute $N=N_{\mathscr{F}}$; and finally construct $\mathscr{K}_{N}$, a finite set of representatives of the $N$ generated members of $\mathscr{K}$.

We claim that $\mathrm{V}\left(\mathscr{K}^{t}\right) \vDash \phi$ iff $\mathrm{V}\left(\mathscr{K}_{N}^{t}\right) \vDash \phi$. Indeed, suppose $\mathrm{V}\left(\mathscr{K}^{t}\right) \not \models \phi$. By Löwenheim's theorem, $\phi$ is false in some countable $\mathbf{C} \in \mathrm{V}\left(\mathscr{K}^{t}\right)$ and, by the theorem of Bulman-Fleming and Werner [1], if $|C|>1$ then $\mathbf{C}$ is isomorphic to $\mathbf{A}(t)$ for some (countable) $\mathbf{A} \leq_{b p} \prod_{x \in X} \mathbf{A}_{x}$ in $\Gamma^{a}(\mathscr{K})$ with $X^{*}$ countable. 
Then Theorem 2.7 and Lemmas 3.4 and 3.5 produce an algebra $\mathbf{A}^{\prime} \in \mathrm{V}\left(\mathscr{K}_{N}\right)$ such that $\mathbf{A}^{\prime}(t) \not \not \phi$, as required.

Now in [6] there is given an algorithm which reduces the decision problem for $\mathrm{V}\left(\mathscr{K}_{N}^{t}\right)$ to the decision problem for Boolean algebras with countably many distinguished ideals (the latter being decidable by [16]), and this algorithm is uniform in the "input parameter" $\mathscr{K}_{N}^{t}$. Hence it can be used to decide $\phi$.

Proof of Lemma 3.4. Let $\mathscr{K}_{0} \subseteq \mathscr{K}$ witness the fact that $\mathscr{K}$ is almost locally homogeneous, and let $\mathrm{L}$ be the type of $\mathscr{K}$. For the purposes of this proof only, we admit the existence of the empty algebra $\varnothing_{\mathrm{L}}$ of type $\mathrm{L}$, which is construed to be a subalgebra of every L-algebra. We stipulate that $\varnothing_{\mathrm{L}} \in \mathscr{K}_{0}$, but continue to assume that $\varnothing_{\mathrm{L}} \notin \mathscr{K}$.

As $\mathrm{L}$ contains no constant symbols, it is possible to find a finite (nonempty) L-algebra $\mathbf{H}$ and a collection $\mathscr{C}$ of subalgebras of $\mathbf{H}$ with these properties:

1. $\mathrm{I}(\mathscr{C})=\mathrm{I}\left(\mathscr{K}_{0}\right)$;

2. For every $\mathbf{B} \in \mathscr{C}, \mathbf{C} \in \mathscr{K}_{0}$ and embedding $\alpha: \mathbf{B} \hookrightarrow \mathbf{C}$ there exists a $\mathbf{D} \in \mathscr{C}$ and an isomorphism $\beta: \mathbf{C} \cong \mathbf{D}$ such that $\mathbf{B} \leq \mathbf{D}$ and $\beta \alpha(b)=b$ for all $b \in B$.

Now let $\mathbf{A} \leq_{b p} \prod_{x \in X} \mathbf{A}_{x}$ be a countable member of $\Gamma^{a}(\mathscr{K})$. To prove the lemma, we shall prove the existence of a family of isomorphisms $\left(\alpha_{x}: \mathbf{A}_{x} \cong\right.$ $\left.\mathbf{A}_{x}^{\prime}\right)_{x \in X}$, where each $\mathbf{A}_{x}^{\prime}$ is in $\mathscr{K} / \mathbf{H}$, such that if $\alpha: A \rightarrow \prod_{x \in X} A_{x}^{\prime}$ is given by $(\alpha a)(x)=\alpha_{x}(a(x))$ and $A^{\prime}=\alpha(A)$, then $\mathbf{A}^{\prime} \in \Gamma_{\text {hom }}^{a}(\mathscr{K} / \mathbf{H})$; and to do this it will suffice to find a family of isomorphisms $\left(\sigma_{x}: \mathbf{B}_{x} \cong \mathbf{E}_{x}\right)_{x \in X}$ satisfying

3. $\mathbf{E}_{x} \in \mathscr{C}$ for all $x \in X$;

4. $\mathbf{B}_{x} \leq \mathbf{A}_{x}$ and $\mathbf{A}_{x}$ is locally homogeneous over $B_{x}$, for all $x \in X$;

5. $\left\{x \in X: a(x) \in B_{x}\right.$ and $\left.\sigma_{x}(a(x))=c\right\}$ is clopen, for all $a \in A$ and $c \in \bigcup \mathscr{\mathscr { C }}$.

Pick an increasing sequence $A^{(0)} \subseteq A^{(1)} \subseteq A^{(2)} \subseteq \cdots$ of finite subuniverses of $\mathbf{A}$ whose union is $A$. For each $n \geq 0$ and $x, y \in X$ let $A_{x}^{(n)}=\operatorname{proj}_{x}\left(A^{(n)}\right)$; enumerate $A^{(n)}$ as $\left\{a_{1}, \ldots, a_{k}\right\}$ and let $\equiv_{n}$ be the equivalence relation on $X$ defined by $x \equiv_{n} y$ iff $\left\langle a_{1}(x), \ldots, a_{k}(x)\right\rangle$ has the same quantifier-free $k$ type in $\mathbf{A}_{x}^{(n)}$ as $\left\langle a_{1}(y), \ldots, a_{k}(y)\right\rangle$ has in $\mathbf{A}_{y}^{(n)}$. (Equivalently, $x \equiv_{n} y$ iff the map $a(x) \mapsto a(y) \quad\left(a \in A^{(n)}\right)$ is a well-defined isomorphism from $\mathbf{A}_{x}^{(n)}$ to $\mathbf{A}_{y}^{(n)}$.) Let $\Pi_{n}$ be the partition of $X$ induced by $\equiv_{n}$. As there are only finitely many quantifier-free $k$-types realized in $\mathscr{K}$, each of which is definable by a quantifier-free formula, $\Pi_{n}$ consists of finitely many clopen sets. We shall find, for each $n \geq 0$ and $N \in \Pi_{n}$, algebras $\mathbf{C}_{N}^{(n)}, \mathbf{D}_{N}^{(n)}$, and $\mathbf{E}_{N}^{(n)}$, an isomorphism $\tau_{N}^{(n)}: \mathbf{D}_{N}^{(n)} \cong \mathbf{E}_{N}^{(n)}$, and a family of isomorphisms $\left(\alpha_{x}^{(n)}: \mathbf{A}_{x}^{(n)} \cong\right.$ $\left.\mathrm{C}_{N}^{(n)}\right)_{x \in N}$ satisfying

6. $\mathbf{D}_{N}^{(n)} \leq \mathbf{C}_{N}^{(n)}$ and $\mathbf{E}_{N}^{(n)} \in \mathscr{C}$

7. $D_{N}^{(n)}$ is a maximal $\mathscr{K}_{0}$-subuniverse of $\mathbf{C}_{N}^{(n)}$;

8. $\alpha_{x}^{(n)}(a(x))=\alpha_{y}^{(n)}(a(y))$ for all $a \in A^{(n)}$ and $x, y \in N$.

Moreover, these algebras and isomorphisms shall be coordinated so that if $n>m, N \in \Pi_{n}, M \in \Pi_{m}$, and $x \in N \subseteq M$ then:

9. $\mathbf{C}_{M}^{(m)} \leq \mathbf{C}_{N}^{(n)}, \mathbf{D}_{M}^{(m)} \leq \mathbf{D}_{N}^{(n)}$, and $\mathbf{E}_{M}^{(m)} \leq \mathbf{E}_{N}^{(n)}$;

10. $\alpha_{x}^{(m)} \subseteq \alpha_{x}^{(n)}$ for all $x \in N$, and $\tau_{M}^{(m)} \subseteq \tau_{N}^{(n)}$;

11. $C_{M}^{(m)} \cap D_{N}^{(n)}=D_{M}^{(m)}$. 
This will be enough, since we can then argue as follows. For each $x \in X$ and $n \geq 0$ pick $N \in \Pi_{n}$ such that $x \in N$ and define $\mathbf{C}_{x}^{(n)}=\mathbf{C}_{N}^{(n)}, \mathbf{D}_{x}^{(n)}=\mathbf{D}_{N}^{(n)}$, and $\mathbf{E}_{x}^{(n)}=\mathbf{E}_{N}^{(n)}$. Also define $B_{x}^{(n)}=\left(\alpha_{x}^{(n)}\right)^{-1}\left(D_{x}^{(n)}\right)$ and let $\sigma_{x}^{(n)}: \mathbf{B}_{x}^{(n)} \cong \mathbf{E}_{x}^{(n)}$ be given by $\sigma_{x}^{(n)}=\left.\tau_{x}^{(n)} \circ \alpha_{x}^{(n)}\right|_{B_{x}^{(n)}}$. Then by construction:

12. $A_{x}^{(m)} \cap B_{x}^{(n)}=B_{x}^{(m)}$ for $m<n$ and

$$
\begin{aligned}
& \mathbf{B}_{x}^{(0)} \leq \mathbf{B}_{x}^{(1)} \leq \mathbf{B}_{x}^{(2)} \leq \cdots \\
& \mathbf{E}_{x}^{(0)} \leq \mathbf{E}_{x}^{(1)} \leq \mathbf{E}_{x}^{(2)} \leq \cdots \\
& \sigma_{x}^{(0)} \subseteq \sigma_{x}^{(1)} \subseteq \sigma_{x}^{(2)} \subseteq \cdots,
\end{aligned}
$$

for each $x \in X$. As each $\mathbf{E}_{x}^{(n)}$ is in $\mathscr{C}$, which is finite, the above sequences must eventually stabilize. For each $x \in X$ let $\mathbf{B}_{x}, \mathbf{E}_{x}$, and $\sigma_{x}$ be the limiting values of the above sequences. We claim that the family $\left(\sigma_{x}: \mathbf{B}_{x} \cong \mathbf{E}_{x}\right)_{x \in X}$ satisfies the conditions (3)-(5) stated above. Certainly condition (3) holds. Since $D_{x}^{(n)}$ is a maximal $\mathscr{K}_{0}$-subuniverse of $\mathbf{C}_{x}^{(n)}, B_{x}^{(n)}$ must be a maximal $\mathscr{K}_{0}$-subuniverse of $\mathbf{A}_{x}^{(n)}$, and hence $\mathbf{A}_{x}^{(n)}$ is locally homogeneous over $B_{x}^{(n)}$ (by our choice of $\left.\mathscr{K}_{0}\right)$. Thus $\mathbf{A}_{x}^{(n)}$ is locally homogeneous over $B_{x}$ for sufficiently large $n$, which implies that $\mathbf{A}_{x}$ is locally homogeneous over $B_{x}$, proving condition (4).

What must be verified is condition (5). Let $a \in A$ be given and pick $m \geq 0$ such that $a \in A^{(m)}$. It follows from condition (12) that for all $x \in X, a(x) \in$ $B_{x}$ iff $a(x) \in B_{x}^{(m)}$. Hence for each $c \in \bigcup \mathscr{C}$ the set $\left\{x \in X: a(x) \in B_{x}\right.$ and $\left.\sigma_{x}(a(x))=c\right\}$ is a union of sets in $\Pi_{m}$, so must be clopen. Hence the family $\left(\sigma_{x}: \mathbf{B}_{x} \cong \mathbf{E}_{x}\right)_{x \in X}$ satisfies conditions (3)-(5) as claimed.

Now we prove the existence of the algebras $\mathbf{C}_{N}^{(n)}, \mathbf{D}_{N}^{(n)}, \mathbf{E}_{N}^{(n)}$, and isomorphisms $\tau_{N}^{(n)}, \alpha_{x}^{(n)}$ satisfying conditions (6)-(11). The proof is by induction on $n$.

First suppose $N \in \Pi_{0}$; pick $x_{0} \in N$ and define $\mathbf{C}_{N}^{(0)}=\mathbf{A}_{x_{0}}^{(0)}$. Let $D_{N}^{(0)}$ be any maximal $\mathscr{K}_{0}$-subuniverse of $\mathbf{A}_{x_{0}}^{(0)}$, and $\tau_{N}^{(0)}$ any isomorphism from $\mathbf{D}_{N}^{(0)}$ to a member $\mathbf{E}_{N}^{(0)}$ of $\mathscr{C}$. For each $x \in N$ define $\alpha_{x}^{(0)}: \mathbf{A}_{x}^{(0)} \cong \mathbf{A}_{x_{0}}^{(0)}$ by $\alpha_{x}^{(0)}(a(x))=$ $a\left(x_{0}\right) \quad\left(a \in A^{(0)}\right)$. This choice of $\mathbf{C}_{N}^{(0)}, \mathbf{D}_{N}^{(0)}, \mathbf{E}_{N}^{(0)}, \tau_{N}^{(0)}$, and $\left(\alpha_{x}^{(0)}\right)_{x \in N}$ certainly satisfies conditions $(6)-(8)$.

Next suppose $k \geq 0$ and we have appropriate algebras and isomorphisms defined for all $n \leq k$, satisfying conditions (6)-(11) for all $m<n \leq k$. Pick $N \in \Pi_{k+1}$ and $M \in \Pi_{k}$ such that $N \subseteq M$. Fix $x_{0} \in N$ and find an algebra $\mathbf{C}_{N}^{(k+1)}$ and an isomorphism $\alpha: \mathbf{A}_{x_{0}}^{(k+1)} \cong \mathbf{C}_{N}^{(k+1)}$ satisfying $\mathbf{C}_{M}^{(k)} \leq \mathbf{C}_{N}^{(k+1)}$ and $\left.\alpha\right|_{A_{x_{0}}^{(k)}}=\alpha_{x_{0}}^{(k)}$. Then define $\alpha_{x}^{(k+1)}: \mathbf{A}_{x}^{(k+1)} \cong \mathbf{C}_{N}^{(k+1)}$ by $\alpha_{x}^{(k+1)}(a(x))=\alpha\left(a\left(x_{0}\right)\right)$ $\left(a \in A^{(k+1)}\right)$ for each $x \in N$. Next, extend $D_{M}^{(k)}$ to a maximal $\mathscr{R}_{0}$-subuniverse $D_{N}^{(k+1)}$ of $\mathbf{C}_{N}^{(k+1)}$ and let $A_{0}=C_{M}^{(k)} \cap D_{N}^{(k+1)}$. Note that $\mathbf{D}_{M}^{(k)} \leq \mathbf{A}_{0} \leq \mathbf{C}_{M}^{(k)}$ and $\mathbf{A}_{0} \in \mathrm{IS}\left(\mathscr{K}_{0}\right)=\mathrm{I}\left(\mathscr{K}_{0}\right)$; hence $A_{0}=D_{M}^{(k)}$ by the inductive hypothesis, condition (7). This verifies condition (11). Finally, pick $\mathbf{E}_{N}^{(k+1)} \in \mathscr{C}$ and $\tau_{N}^{(k+1)}: \mathbf{D}_{N}^{(k+1)} \cong \mathbf{E}_{N}^{(k+1)}$ so that $\mathbf{E}_{M}^{(k)} \leq \mathbf{E}_{N}^{(k+1)}$ and $\left.\tau_{N}^{(k+1)}\right|_{D_{M}^{(k)}}=\tau_{M}^{(k)}$. (This can be done by our choice of $\mathbf{H}$ and $\mathscr{C}$.) The reader can check that this choice of $\mathbf{C}_{N}^{(k+1)}, \mathbf{D}_{N}^{(k+1)}, \mathbf{E}_{N}^{(k+1)}, \tau_{N}^{(k+1)}$, and $\left(\alpha_{x}^{(k+1)}\right)_{x \in N}$ satisfies $(6)-(10)$. 
Proof of Lemma 3.5. Let $\mathscr{F}=\left\{\mathbf{B}_{1}, \ldots, \mathbf{B}_{k}\right\}$ and suppose each $\mathbf{B}_{i}$ is $n_{i}$ generated. Let $N_{\mathscr{F}}=n_{1}+\cdots+n_{k}+1$. We claim that this works. Indeed, suppose $\mathbf{A} \leq_{b p} \prod_{x \in X} \mathbf{A}_{x}$ is in $\Gamma_{\text {hom }}^{a}(\mathscr{K} / \mathbf{H})$ with $X^{*}$ countable; fix $c \in A$. For each $i=1, \ldots, k$ pick a clopen partition of the open set $U_{\mathbf{B}_{i}}$ (this is possible by the countability of $X^{*}$ ), and further refine this partition (let $\Pi_{i}$ denote the refined partition) by requiring that for each $N \in \Pi_{i}$ there exist $b_{1}, \ldots, b_{n_{i}} \in A$ such that for all $x \in N$, the set $\left\{b_{1}(x), \ldots, b_{n_{i}}(x)\right\}$ generates a subalgebra of $\mathbf{A}_{x} \mathbf{H}$-isomorphic to $\mathbf{B}_{i}$. With $N$ fixed and for $j=1, \ldots, n_{i}$ define $a_{j}^{i, N}=\left.\left.b_{j}\right|_{N} \cup c\right|_{X \backslash N}$, and put

$$
\Delta=\left\{a_{j}^{i, N}: 1 \leq i \leq k, N \in \Pi_{i}, 1 \leq j \leq n_{i}\right\}
$$

(or $\Delta=\{c\}$ if $\mathscr{F}=\varnothing$ ). Finally let $A^{\prime}$ be the closure under patchwork of $\operatorname{Sg}_{\mathbf{A}}(\Delta)$. Clearly $\mathbf{A}^{\prime} \leq_{b p} \prod_{x \in X} \mathbf{A}_{x}^{\prime}$ where $A_{x}^{\prime}=\operatorname{Sg}_{\mathbf{A}_{x}}(\{a(x): a \in \Delta\})$ for each $x \in X$. By Claim 2.2(i), $\mathbf{A}_{x}^{\prime}$ is locally homogeneous over $A_{x}^{\prime} \cap H$, so $\mathbf{A}_{x}^{\prime} \in \Gamma_{\text {hom }}^{a}(\mathscr{K} / \mathbf{H})$. This establishes (i). (ii) and (iii) follow immediately from the construction of $\mathbf{A}^{\prime}$.

\section{A SEMANTIC EMBEDDING}

Let $\mathbf{A}=\langle A, f\rangle$ be the mono-unary algebra defined by $A=\{-1\} \cup \omega$ and $f(n)=\max (n, 0)$, and let $\mathscr{K}_{1}$ be the smallest universal class containing $\mathbf{A}$. $(\mathscr{K} 1$ was presented in [4] as the simplest class $\mathscr{K}$ for which the decidability of $\mathrm{V}\left(\mathscr{K}^{t}\right)$ was unknown.) Let $\mathbf{S}$ be the subalgebra of $\mathbf{A}$ whose universe is $\omega$. Clearly the automorphism group of $\mathbf{S}$ is transitive while the element $0 \in S$ is fixed by every automorphism of $\mathbf{A}$. These facts alone are enough to prove that $\mathscr{K}_{1}$ is not almost locally homogeneous (exercise, or see the proof of Theorem 5.3), so the methods of the previous section cannot be applied.

The following lemma contains a semantic embedding which grew out of our study of $\mathscr{K}_{1}$. The lemma is sufficient for the purposes of this paper, and will likely have further applications. The statement of the lemma is similar in form to, and was motivated by, Lemma 2.1 in [4]; and though it appears that neither lemma generalizes the other, ours is an improvement with respect to two subtle details, explained below.

Lemma 4.1. Suppose $\mathbf{A}$ is an algebra of type $\mathbf{L}, \mathbf{S}$ is a subalgebra of $\mathbf{A}$, and $\mu(\vec{x})$ and $\tau(\vec{x})$ are first-order L-formulas. Define $M=\left.\mu^{\mathbf{A}}\right|_{S}$ and $T=\left.M \cap \tau^{\mathbf{A}}\right|_{S}$. Suppose also that $\equiv$ is an equivalence relation on $M$ such that $T$ is a union of 三-classes, and define

Aut $_{M, \equiv \mathbf{S}}=\{\sigma \in$ Aut $\mathbf{S}: \sigma(M)=M$ and for all $a, b, \in M$,

$$
a \equiv b \text { iff } \sigma(a) \equiv \sigma(b)\} .
$$

If:

(1) $M$ contains infinitely many $\equiv$-classes while $T$ contains only finitely many $\equiv$-classes;

(2) $\bigcup\left\{\sigma(T): \sigma \in \mathrm{Aut}_{M, \equiv} \mathbf{S}\right\}=M$;

(3) there is an L-formula $\psi(\vec{z})$ such that $\psi^{\mathbf{A}} \neq \varnothing$ but $\left.\psi^{\mathbf{A}}\right|_{S}=\varnothing$;

then the class $\operatorname{SP}\left(\mathbf{A}^{t}\right)$ is hereditarily undecidable. 
Before giving the proof, we wish to compare our lemma with the corresponding lemma in [4]. The chief improvements are $^{3}$ : one.

- In [4] it is required that $T$ contain at least two $\equiv$-classes; we require only

- Our assumption (2) replaces the assumption in [4] of the existence of a complete set of representatives for the $\equiv$-classes in $M$, which generates $\mathbf{S}$ and is "independent" in a very strong sense; for example, Aut $\mathbf{S}$ must act $n$-fold transitively on this set for all $n$.

It is the first improvement which we exploit in this paper. For an illustration of the value of the second improvement, see [20].

Proof. First, note that we can assume that $\tau^{\mathbf{A}} \subseteq \mu^{\mathbf{A}}$ and hence $\left.\tau^{\mathbf{A}}\right|_{S}=T$. Next, pick a subset $\Gamma \subseteq \operatorname{Aut}_{M}, \equiv \mathbf{S}$ such that $\{\sigma(T): \sigma \in \Gamma\}=\left\{\sigma(T): \sigma \in\right.$ Aut $\left._{M}, \equiv \mathbf{S}\right\}$ and for $\sigma_{1}, \sigma_{2} \in \Gamma, \sigma_{1}(T)=\sigma_{2}(T)$ iff $\sigma_{1}=\sigma_{2}$. Now we dispose of the equivalence relation $\equiv$; its only role is to ensure that the following conditions are met:

(4) $\Gamma$ is infinite;

(5) For all $\sigma_{1}, \sigma_{2} \in \Gamma, M \nsubseteq \sigma_{1}(T) \cup \sigma_{2}(T)$;

(6) There is a positive integer $k$ such that for all $\sigma \in \Gamma$ there is a subset $T_{0} \subseteq \sigma(T)$ with $\left|T_{0}\right|=k$ and such that for all $\sigma^{\prime} \in \Gamma, T_{0} \subseteq \sigma^{\prime}(T)$ iff $\sigma^{\prime}=\sigma$.

Let $\kappa=|\Gamma|$. We shall semantically embed into $\mathrm{SP}\left(\mathbf{A}^{t}\right)$ the class of all graphs of cardinality $\kappa$ in which every vertex belongs to an edge. Let $\mathscr{G}=(V, E)$ be such a graph. For each $v \in V$ and $e \in E$ let $v^{*}=\{v\} \times \omega$ and $e^{*}=\{e\} \times \omega$, and put $V^{*}=\bigcup\left\{v^{*}: v \in V\right\}$ and $E^{*}=\bigcup\left\{e^{*}: e \in E\right\}$. Next define

$$
\begin{aligned}
& Y=\bigcup\left\{e^{*} \times v^{*}: v \in e \in E\right\}, \\
& X=Y \cup E^{*} \cup\{\infty\} .
\end{aligned}
$$

Define a Boolean algebra of subsets of $X$ as follows. For each $x=(e, i) \in$ $E^{*}$ let

$$
\begin{aligned}
N_{x} & =\{x\} \cup\left(\left[\{x\} \times V^{*}\right] \cap Y\right) \\
& =\{x\} \cup\left\{(x, y): y \in v^{*} \cup w^{*}\right\} \quad \text { where } e=\{v, w\} .
\end{aligned}
$$

For each finite or cofinite subset $A$ of $E^{*}$ define

$$
N_{A}= \begin{cases}\bigcup\left\{N_{x}: x \in A\right\} & \text { if } A \text { is finite, } \\ \bigcup\left\{N_{x}: x \in A\right\} \cup\{\infty\} & \text { if } A \text { is cofinite. }\end{cases}
$$

Finally, let

$$
\begin{aligned}
\mathscr{B}_{0} & =\left\{N_{A}: A \text { is a finite or cofinite subset of } E^{*}\right\}, \\
\mathscr{B} & =\left\{N \oplus F: N \in \mathscr{B}_{0} \text { and } F \text { is a finite subset of } Y\right\} .
\end{aligned}
$$

Clearly $\mathscr{B}$ is a Boolean subalgebra of $\operatorname{Su}(X)$. In fact, $\mathscr{B}$ is a basis for a compact Hausdorff topology $\mathscr{T}$ on $X$. Thus $(X, \mathscr{T})$ is a Boolean space and $\mathscr{B}$ is its Boolean algebra of clopen sets. Under this topology, $Y$ is precisely the set of isolated points of $X$.

\footnotetext{
${ }^{3}$ In [4] it is also required that $\mu$ and $\tau$ have only one free variable, and that the relation $\equiv$ be definable in $\mathbf{A}$. On the other hand, [4] does not require our condition (3), and weakens our assumption (1) to require only that $M \backslash T$ contain infinitely many $\equiv$-classes.
} 
For each $v \in V$ let $U_{v}=\left(\bigcup\left\{e^{*}: v \in e \in E\right\}\right) \times v^{*}$. Note that each $U_{v}$ is open (and nonempty, by our assumptions on $\mathscr{G}$ ); that the $U_{v}$ s partition $Y$; and that for $e \in E, U_{v} \cap N_{e^{*}}=e^{*} \times v^{*}$ if $v \in e$ while $U_{v} \cap N_{e^{*}}=\varnothing$ otherwise.

We define an algebra in $\operatorname{ISP}\left(\mathbf{A}^{t}\right)$ as follows. First pick a bijection between $V$ and $\Gamma$; denote it by $v \mapsto \sigma_{v}$. Next, pick an algebra $\mathbf{P}$, a family of subalgebras $\left(\mathbf{A}_{v}\right)_{v \in V}$ of $\mathbf{P}$, and a family of isomorphisms $\left(\hat{\sigma}_{v}: \mathbf{A} \cong \mathbf{A}_{v}\right)_{v \in V}$ satisfying:

1. $\mathbf{S} \leq \mathbf{A}_{v}$ for all $v \in V$;

2. $\left.\hat{\sigma}_{v}\right|_{S}=\sigma_{v}$ for all $v \in V$;

3. For all $v, w \in V$ with $v \neq w, A_{v} \cap A_{w}=S$.

Note that $\left.\mu^{\mathbf{A}_{v}}\right|_{S}=M$ and $\left.\tau^{\mathbf{A}_{v}}\right|_{S}=\sigma_{v}(T)$ for each $v \in V$.

Finally, define $\mathbf{C}$ to be the subalgebra of $\left(\mathbf{P}^{t}\right)^{X}$ consisting of the continuous functions $a: X \rightarrow P$ (where $P$ is given the discrete topology) which satisfy $a\left(U_{v}\right) \subseteq A_{v}$ for all $v \in V$ (and consequently $\left.a(X \backslash Y) \subseteq S\right)$. Note that each $a \in C$ is finite valued, its values lie in $\bigcup\left\{A_{v}: v \in V\right\}$ and have clopen support, and its values lie in $S$ except on a finite subset of $Y$. Examples of elements in $C$ are the constant functions $c_{s}: X \rightarrow\{s\}$ for each $s \in S$. It is easy to verify that $\mathbf{C}$ is a Boolean product of algebras $\left(\mathbf{C}_{x}\right)_{x \in X}$, where $\mathbf{C}_{x}=\mathbf{A}_{v}^{t}$ if $x \in U_{v}$, and $\mathbf{C}_{x}=\mathbf{S}^{t}$ if $x \in X \backslash Y$. Hence $\mathbf{C} \in \operatorname{ISP}\left(\mathbf{A}^{t}\right)$.

Now we shall show how $\mathscr{G}$ can be interpreted in $\mathbf{C}$. In what follows, let us say that a structure (obtained in some way from $\mathscr{G}$ ) is definable in $\mathbf{C}$ if there are first-order $\mathrm{L}(t)$-formulas which interpret the structure in $\mathbf{C}$ in the usual way (see [15] or [3]). In particular, the formulas must not depend on the particular choice of $\mathscr{G}$. We shall also use the following standard notation: if $\phi\left(z_{1}, \ldots, z_{n}\right)$ is an $\mathrm{L}(t)$-formula and $a_{1}, \ldots, a_{n} \in C$, then $\llbracket \phi(\vec{a}) \rrbracket$ denotes the set $\left\{x \in X: \mathbf{C}_{x} \vDash \phi\left(a_{1}(x), \ldots, a_{n}(x)\right)\right\}$.

Claim 4.2. $\mathscr{B}$ is definable in $\mathbf{C}$.

Proof. Since $C$ contains two distinct constant functions and is closed under patchwork, the elements of $\mathscr{B}$ (that is, the clopen sets of $X$ ) are definable as 'nonequalizer sets' $\llbracket a \neq b \rrbracket$. Inclusion and equality of nonequalizer sets can be defined using the switching term $s(x, y, z, w): \llbracket a \neq b \rrbracket \subseteq \llbracket c \neq d \rrbracket$ iff $s^{\mathbf{C}}(c, d, a, b)=b$.

Claim 4.3. For every first-order $\mathrm{L}(t)$-formula $\phi(\vec{x})$ there is a first-order $\mathrm{L}(t)$ formula $\phi^{*}(\vec{x})$ such that for any $\vec{a}$ in $C$ we have

$$
Y \subseteq \llbracket \phi(\vec{a}) \rrbracket \quad \Leftrightarrow \quad \mathbf{C} \vDash \phi^{*}(\vec{a}) .
$$

Moreover, $\phi^{*}$ is independent of the original graph $\mathscr{G}$.

Proof. This was essentially proved in Claim 2.3 of [4]; the definition of $\phi^{*}$ given there works here as well. The only fact that matters is that $Y$ is the set of those isolated points $x \in X$ satisfying $\left|C_{x}\right|>1$.

Claim 4.4. The finite subsets of $Y$ are definable in $\mathbf{C}$.

Proof. We shall show that the finite subsets of $Y$ are precisely the clopen sets $\llbracket a \neq b \rrbracket$ satisfying $Y \subseteq \llbracket a \neq b \rightarrow \psi(\vec{c}) \rrbracket$ for some $\vec{c}$ in $C$. The claim will then follow from Claim 4.3.

Suppose first that $F$ is a finite subset of $Y$. Then $F$ is clopen and so is of the form $\llbracket a \neq b \rrbracket$ for some $a, b \in C$. For each $x \in F, \mathbf{C}_{x} \cong \mathbf{A}^{t}$ so by hypothesis (3) of Lemma 4.1 it is possible to pick $\vec{c}_{x}$ in $C_{x}$ such that $\mathbf{C}_{x} \vDash \psi\left(\vec{c}_{x}\right)$. As 
$\{x\}$ is clopen for each $x \in F$, and as $C$ is closed under patchwork, it follows that there exist $\vec{c}$ in $C$ such that $\vec{c}(x)=\vec{c}_{x}$ for all $x \in F$. Then $F \subseteq \llbracket \psi(\vec{c}) \rrbracket$ and hence $\llbracket a \neq b \rightarrow \psi(\vec{c}) \rrbracket=X$.

Conversely, suppose $F=\llbracket a \neq b \rrbracket$ is a clopen set and $Y \subseteq \llbracket a \neq b \rightarrow \psi(\vec{c}) \rrbracket$, or equivalently, $F \cap Y \subseteq \llbracket \psi(\vec{c}) \rrbracket$. Since $\vec{c}(x)$ is in $S$ for all $x \in X$ except on some finite subset of $Y$, hypothesis (3) of Lemma 4.1 implies that $\llbracket \psi(\vec{c}) \rrbracket \cap Y$ is finite. It follows that $F \cap Y$ is a finite subset of $Y$. But $F$ is in $\mathscr{B}$; hence $F \subseteq Y$, which proves the claim.

Note that it follows from the previous claim that the relation $N \stackrel{\text { ae }}{=} N^{\prime}$, defined on $\mathscr{B}$ to mean $N^{\prime}=N \oplus F$ for some finite subset $F$ of $Y$, is definable in $\mathbf{C}$, as is the subset $\left\{N \in \mathscr{B}: N \stackrel{\text { ae }}{=} N_{x}\right.$ for some $\left.x \in E^{*}\right\}$.

\section{Definition 4.5.}

(1) $R=\{\vec{a}$ in $C: Y \subseteq \llbracket \mu(\vec{a}) \rrbracket\}$.

(2) For $\vec{a} \in R, \mathscr{O}_{\vec{a}}=\llbracket \tau(\vec{a}) \rrbracket \cap Y$.

$R$ is definable in $\mathbf{C}$ by Claim 4.3. Note that each $\mathscr{O}_{\vec{a}}$ is an open set (since it is a subset of $Y$ ).

Claim 4.6. The following predicates are definable in $\mathbf{C}\left(\right.$ for $\vec{a}, \vec{b}, \vec{a}_{1}, \ldots, \vec{a}_{k} \in$ $R$ and $N \in \mathscr{B})$ :

(i) $N \subseteq \mathscr{O}_{\vec{a}}$.

(ii) $\mathscr{O}_{\vec{a}} \subseteq N$.

(iii) (For fixed $k$ ): $\mathscr{O}_{\vec{a}_{1}} \cap \cdots \cap \mathscr{O}_{\vec{a}_{k}} \subseteq N$.

(iv) (For fixed $k$ ): $\mathscr{O}_{\vec{a}_{1}} \cap \cdots \cap \mathscr{O}_{\vec{a}_{k}} \cap N \subseteq \mathscr{O}_{\vec{b}}$.

Proof. $N \subseteq \mathscr{O}_{\vec{a}}$ iff $N$ is a finite subset of $Y$ and $N \subseteq \llbracket \tau(\vec{a}) \rrbracket$. The latter condition is equivalent to $Y \subseteq \llbracket b \neq c \rightarrow \tau(\vec{a}) \rrbracket$, where $N=\llbracket b \neq c \rrbracket$, and this is definable by Claim 4.3. This proves (i). (ii)-(iv) follow from (i) and the fact that each $\mathscr{O}_{\vec{a}}$ is open.

Definition 4.7. For $\vec{a} \in R$, $\operatorname{supp}(\vec{a})=\left\{v \in V: \vec{a}(\infty) \in \sigma_{v}(T)\right\}$.

Claim 4.8. For any $\vec{a} \in R$ :

(i) $\operatorname{supp}(\vec{a}) \neq \varnothing$.

(ii) If $v \in V$ and $N$ is a clopen neighborhood of $\infty$ on which $\vec{a}$ is constant, then the following are equivalent

(1) $v \in \operatorname{supp}(\vec{a})$.

(2) $\infty \in \overline{\mathscr{O}_{\vec{a}} \cap U_{v}}$. (Here and elsewhere, $\bar{W}$ denotes the topological closure of $W$.)

(3) $N \cap U_{v} \subseteq \mathscr{O}_{\vec{a}}$.

(4) $N \cap U_{v} \cap \mathscr{O}_{\vec{a}} \neq \varnothing$.

Proof. (i) Define $\vec{a}_{0}=\vec{a}(\infty)$. It will suffice, by hypothesis (2) of Lemma 4.1, to show that $\vec{a}_{0} \in M$. Clearly $\vec{a}_{0}$ is in $S$ (as $\mathbf{C}_{\infty}=\mathbf{S}^{t}$ ). Let $N=\{x \in X: \vec{a}(x)=$ $\left.\vec{a}_{0}\right\} . \quad N$ is a clopen neighborhood of $\infty$, so $N \cap Y \neq \varnothing$. Pick $x \in N \cap Y$; as $Y \subseteq \llbracket \mu(\vec{a}) \rrbracket$ it follows that $\left.\vec{a}_{0} \in \mu^{\mathrm{C}_{x}}\right|_{S}=M$ (see the remark before the definition of $\mathbf{C}$ ), as desired.

(ii) Let $N$ be a clopen neighborhood of $\infty$ on which $\vec{a}$ is constant. If $v \in \operatorname{supp}(\vec{a})$, i.e., $\vec{a}(\infty) \in \sigma_{v}(T)=\left.\tau^{\mathbf{A}_{v}}\right|_{S}$, then $N \cap U_{v} \subseteq \llbracket \tau(\vec{a}) \rrbracket$ (since $\mathbf{C}_{x}=\mathbf{A}_{v}^{t}$ 
for all $\left.x \in U_{v}\right)$; hence $N \cap U_{v} \subseteq \mathscr{O}_{\vec{a}}$. On the other hand, if $v \notin \operatorname{supp}(\vec{a})$ then $N \cap U_{v} \cap \mathscr{O}_{\vec{a}}=\varnothing$ by the same reasoning. This proves the equivalence of (1), (3), and (4). Certainly (2) implies (4). Conversely, suppose (1) is true, and hence (4) holds for all clopen neighborhoods $N^{\prime}$ of $\infty$ on which $\vec{a}$ is constant. It is easy to see that for every clopen neighborhood $N$ of $\infty$ there is a clopen subset $N^{\prime}$, also a neighborhood of $\infty$, on which $\vec{a}$ is constant. Then (2) follows.

Claim 4.9. The ultrafilter $U_{\infty}$ of clopen neighborhoods of $\infty$ is definable in $\mathbf{C}$. Proof. The definition is: $N \in \mathrm{U}_{\infty}$ iff there exists $\vec{a} \in R$ such that $\mathscr{O}_{\vec{a}} \subseteq N$. (The latter condition is definable in $\mathbf{C}$ by (ii) of Claim 4.6.) It follows from Claim 4.8 that if $\mathscr{O}_{\vec{a}} \subseteq N$ then $\infty \in \overline{\mathscr{O}_{\vec{a}}} \subseteq N$. Conversely, if $\infty \in N$ then $N \supseteq N_{A}$ for some cofinite subset $A$ of $E^{*}$. Consider $(e, i) \in E^{*} \backslash A$ where $e=\{v, w\}$. By condition (5) at the beginning of the proof of Lemma 4.1, $M \nsubseteq \sigma_{v}(T) \cup \sigma_{w}(T)$. Pick $\vec{a}_{(e, i)} \in M \backslash\left(\sigma_{v}(T) \cup \sigma_{w}(T)\right)=M \backslash\left(\tau^{A_{v}} \cup \tau^{A_{w}}\right)$, and note that for all $x \in N_{(e, i)} \cap Y, \vec{a}_{(e, i)} \in \mu^{\mathbf{C}_{x}} \backslash \tau^{\mathbf{C}_{x}} \quad$ (as $\mathbf{C}_{x}$ is either $\mathbf{A}_{v}^{t}$ or $\left.\mathbf{A}_{w}^{t}\right)$. Since the constant maps $c_{s}(s \in S)$ are in $C$, each $N_{(e, i)}$ is clopen, and $C$ is closed under patchwork, there is a tuple $\vec{a}$ in $C$ such that for each $(e, i) \in E^{*} \backslash A, \vec{a}(x)=\vec{a}_{(e, i)}$ for all $x \in N_{(e, i)}$, and $\vec{a}(x) \in M$ for all $x \in N_{A}$. It follows that $\vec{a} \in R$ and $\mathscr{O}_{\vec{a}} \subseteq N_{A} \subseteq N$ as desired.

Definition 4.10. Let $k$ be fixed as in condition (6) at the beginning of the proof of Lemma 4.1. For $\vec{a}_{1}, \ldots, \vec{a}_{k} \in R$,

$$
\operatorname{supp}_{k}\left(\vec{a}_{1}, \ldots, \vec{a}_{k}\right)=\operatorname{supp}\left(\vec{a}_{1}\right) \cap \cdots \cap \operatorname{supp}\left(\vec{a}_{k}\right) \text {. }
$$

\section{Claim 4.11.}

(i) For every $v \in V$ there exists $\vec{a}_{1}, \ldots, \vec{a}_{k} \in R$ such that $\operatorname{supp}_{k}\left(\vec{a}_{1}, \ldots, \vec{a}_{k}\right)$ $=\{v\}$.

(ii) The following predicates are definable in $\mathbf{C}$ (with $\left.\vec{a}_{i}, \vec{b}_{i} \in R\right)$ :

(1) $\operatorname{supp}_{k}\left(\vec{a}_{1}, \ldots, \vec{a}_{k}\right) \subseteq \operatorname{supp}_{k}\left(\vec{b}_{1}, \ldots, \vec{b}_{k}\right)$,

(2) $\operatorname{supp}_{k}\left(\vec{a}_{1}, \ldots, \vec{a}_{k}\right)=\operatorname{supp}_{k}\left(\vec{b}_{1}, \ldots, \vec{b}_{k}\right)$,

(3) $\operatorname{supp}_{k}\left(\vec{a}_{1}, \ldots, \vec{a}_{k}\right)=\{v\}$ for some $v \in V$.

Proof. (i) By condition (6) at the beginning of the proof of Lemma 4.1, there exist $\vec{a}_{1}, \ldots, \vec{a}_{k} \in \sigma_{v}(T)$ such that for all $w \in V,\left\{\vec{a}_{1}, \ldots, \vec{a}_{k}\right\} \subseteq \sigma_{w}(T)$ iff $w=v$. Consider the corresponding tuples of constant maps $\vec{c}_{\vec{a}_{1}}, \ldots, \vec{c}_{\vec{a}_{k}}$ in $C$. As $\vec{c}_{\vec{a}_{i}}(x)=\vec{a}_{i} \in M \subseteq \mu^{\mathbf{C}_{x}}$ for all $x \in Y$, it follows that $\vec{c}_{\vec{a}_{i}} \in R$ for each $i$. It is now easy to check that $\operatorname{supp}_{k}\left(\vec{c}_{\vec{a}_{1}}, \ldots, \vec{c}_{\vec{a}_{k}}\right)=\{v\}$.

(ii) By (i) it will be enough to prove that, for $\vec{a}_{1}, \ldots, \vec{a}_{k}, \vec{b} \in R$, the predicate “ $\operatorname{supp}_{k}\left(\vec{a}_{1}, \ldots, \vec{a}_{k}\right) \subseteq \operatorname{supp}(\vec{b})$ " is definable in $\mathbf{C}$. We claim that $\operatorname{supp}_{k}\left(\vec{a}_{1}, \ldots, \vec{a}_{k}\right) \subseteq \operatorname{supp}(\vec{b})$ iff $\mathscr{O}_{\vec{a}_{1}} \cap \cdots \cap \mathscr{O}_{\vec{a}_{k}} \cap N \subseteq \mathscr{O}_{\vec{b}}$ for some $N \in U_{\infty}$. (The latter predicate is definable in $C$ by Claims 4.6(iv) and 4.9.) Indeed, suppose first that $\operatorname{supp}_{k}\left(\vec{a}_{1}, \ldots, \vec{a}_{k}\right) \subseteq \operatorname{supp}(\vec{b})$. Pick $N \in U_{\infty}$ on which each $\vec{a}_{i}$ and $\vec{b}$ is constant, and let $x \in \mathscr{O}_{\vec{a}_{1}} \cap \cdots \cap \mathscr{O}_{\vec{a}_{k}} \cap N$. Let $v \in V$ be such that $x \in U_{v}$. Then $N \cap U_{v} \cap \mathscr{O}_{\vec{a}_{i}} \neq \varnothing$ for each $i$, so by Claim 4.8(ii), $v \in \operatorname{supp}_{k}\left(\vec{a}_{1}, \ldots, \vec{a}_{k}\right)$. Hence $v \in \operatorname{supp}(\vec{b})$, so again by Claim 4.8(ii), $N \cap U_{v} \subseteq \mathscr{O}_{\vec{b}}$ and hence $x \in \mathscr{O}_{\vec{b}}$. This proves $\mathscr{O}_{\vec{a}_{1}} \cap \cdots \cap \mathscr{O}_{\vec{a}_{k}} \cap N \subset \mathscr{O}_{\vec{b}}$. Conversely, assume that this inclusion holds for some $N \in \mathrm{U}_{\infty}$. We may replace $N$ 
by a smaller clopen set if necessary so that each $\vec{a}_{i}$ and $\vec{b}$ is constant on $N$. If $v \in \operatorname{supp}_{k}\left(\vec{a}_{1}, \ldots, \vec{a}_{k}\right)$ then by Claim 4.8(ii), $N \cap U_{v} \subseteq \mathscr{O}_{\vec{a}_{1}} \cap \cdots \cap \mathscr{O}_{\vec{a}_{k}}$. It follows from our assumption that $N \cap U_{v} \subseteq \mathscr{O}_{\vec{b}}$, and hence $v \in \operatorname{supp}(\vec{b})$. This proves $\operatorname{supp}_{k}\left(\vec{a}_{1}, \ldots, \vec{a}_{k}\right) \subseteq \operatorname{supp}(\vec{b})$.

Claim 4.12. Suppose $\operatorname{supp}_{k}\left(\vec{a}_{1}, \ldots, \vec{a}_{k}\right)=\{v\}$ and $\operatorname{supp}_{k}\left(\vec{b}_{1}, \ldots, \vec{b}_{k}\right)=\{w\}$ with $v \neq w$. Then $\{v, w\} \in E$ iff for all $\vec{c}_{1}, \ldots, \vec{c}_{k}, \vec{d}_{1}, \ldots, \vec{d}_{k} \in R$ with $\operatorname{supp}_{k}\left(\vec{c}_{1}, \ldots, \vec{c}_{k}\right)=\{v\}$ and $\operatorname{supp}_{k}\left(\vec{d}_{1}, \ldots, \vec{d}_{k}\right)=\{w\}$,

$$
\overline{\mathscr{O}_{\vec{c}_{1}} \cap \cdots \cap \mathscr{O}_{\vec{c}_{k}}} \cap \overline{\mathscr{O}_{\vec{d}_{1}} \cap \cdots \cap \mathscr{O}_{\vec{d}_{k}}} \cap E^{*} \neq \varnothing \text {. }
$$

Proof. First suppose $\{v, w\}=e \in E$ and $\vec{c}_{1}, \ldots, \vec{c}_{k}, \vec{d}_{1}, \ldots, \vec{d}_{k}$ are given as above. Pick a cofinite subset $A$ of $E^{*}$ such that each $\vec{c}_{i}$ and $\vec{d}_{i}$ is constant on $N_{A}$. By Claim 4.8(ii), we have

$$
N_{A} \cap U_{v} \subseteq \mathscr{O}_{\vec{c}_{1}} \cap \cdots \cap \mathscr{O}_{\vec{c}_{k}}
$$

and

$$
N_{A} \cap U_{w} \subseteq \mathscr{O}_{\vec{d}_{1}} \cap \cdots \cap \mathscr{O}_{\vec{d}_{k}}
$$

Since $A$ is cofinite, there is an $i \in \omega$ such that $(e, i) \in A$. Then $(e, i) \in$ $\overline{N_{A} \cap U_{v}} \cap \overline{N_{A} \cap U_{w}}$, which proves one direction of the claim.

Conversely, suppose $\{v, w\} \notin E$. In the proof of Claim 4.11(i) it was shown that it is possible to pick tuples of constant maps $\vec{c}_{i}, \vec{d}_{i} \in R$ such that $\operatorname{supp}_{k}\left(\vec{c}_{1}, \ldots, \vec{c}_{k}\right)=\{v\}$ and $\operatorname{supp}_{k}\left(\vec{d}_{1}, \ldots, \vec{d}_{k}\right)=\{w\}$. Since these maps are constant on $X$, Claim 4.8(ii) implies

$$
\mathscr{O}_{\vec{c}_{1}} \cap \cdots \cap \mathscr{O}_{\vec{c}_{k}}=U_{v}
$$

and

$$
\mathscr{O}_{\vec{d}_{1}} \cap \cdots \cap \mathscr{O}_{\vec{d}_{k}}=U_{w} \text {. }
$$

But $\{v, w\} \notin E$ implies $\bar{U}_{v} \cap \bar{U}_{w} \cap E^{*}=\varnothing$, as desired.

Claim 4.13. The predicate

$$
\overline{\mathscr{O}_{\overrightarrow{c_{1}}} \cap \cdots \cap \mathscr{O}_{\overrightarrow{c_{k}}}} \cap \overline{\mathscr{O}_{\overrightarrow{d_{1}}} \cap \cdots \cap \mathscr{O}_{\overrightarrow{d_{k}}}} \cap E^{*} \neq \varnothing
$$

is definable in $\mathbf{C}$.

Proof. $\overline{\mathscr{O}_{\vec{c}_{1}} \cap \cdots \cap \mathscr{O}_{\overrightarrow{c_{k}}}} \cap \overline{\mathscr{O}_{\vec{d}_{1}} \cap \cdots \cap \mathscr{O}_{\overrightarrow{d_{k}}}} \cap E^{*} \neq \varnothing$ iff there is an $N \stackrel{\text { ae }}{=} N_{x}$ for some $x \in E^{*}$ such that both $N \cap \mathscr{O}_{\vec{c}_{1}} \cap \cdots \cap \mathscr{O}_{\vec{c}_{k}}$ and $N \cap \mathscr{O}_{\vec{d}_{1}} \cap \cdots \cap \mathscr{O}_{\vec{d}_{k}}$ are infinite. Now $N \cap \mathscr{O}_{\vec{c}_{1}} \cap \cdots \cap \mathscr{O}_{\vec{c}_{k}}$ is infinite iff there does not exist an $N_{1} \stackrel{\text { ae }}{=} N$ such that $N_{1} \cap \mathscr{O}_{\vec{c}_{1}} \cap \cdots \cap \mathscr{O}_{\vec{c}_{k}}=\varnothing$; and this last clause is definable in $\mathbf{C}$ by Claim 4.6(iii).

Now we can complete the proof of Lemma 4.1. We have shown that there exist $\mathrm{L}(t)$-formulas $\operatorname{Vert}\left(\vec{x}_{1}, \ldots, \vec{x}_{k}\right), \operatorname{Eq}\left(\vec{x}_{1}, \ldots, \vec{x}_{k}, \vec{y}_{1}, \ldots, \vec{y}_{k}\right)$, and $\operatorname{Edge}\left(\vec{x}_{1}, \ldots, \vec{x}_{k}, \vec{y}_{1}, \ldots, \vec{y}_{k}\right)$, which are independent of the choice of $\mathscr{G}$ and which assert the following in $\mathbf{C}$

$$
\begin{aligned}
\operatorname{Vert}\left(\vec{x}_{1}, \ldots, \vec{x}_{k}\right) & \Leftrightarrow \quad \vec{x}_{1}, \ldots, \vec{x}_{k} \in R \text { and } \operatorname{supp}_{k}\left(\vec{x}_{1}, \ldots, \vec{x}_{k}\right)=\{v\} \\
& \text { for some } v \in V ;
\end{aligned}
$$




$$
\mathrm{Eq}\left(\vec{x}_{1}, \ldots, \vec{x}_{k}, \vec{y}_{1}, \ldots, \vec{y}_{k}\right) \quad \Leftrightarrow \quad \operatorname{Vert}\left(\vec{x}_{1}, \ldots, \vec{x}_{k}\right) \text { and } \operatorname{Vert}\left(\vec{y}_{1}, \ldots, \vec{y}_{k}\right)
$$

$\operatorname{Edge}\left(\vec{x}_{1}, \ldots, \vec{x}_{k}, \vec{y}_{1}, \ldots, \vec{y}_{k}\right) \Leftrightarrow \operatorname{Vert}\left(\vec{x}_{1}, \ldots, \vec{x}_{k}\right)$ and $\operatorname{Vert}\left(\vec{y}_{1}, \ldots, \vec{y}_{k}\right)$ and not $\operatorname{Eq}\left(\vec{x}_{1}, \ldots, \vec{x}_{k}, \vec{y}_{1}, \ldots, \vec{y}_{k}\right)$, and for all $\vec{z}_{1}, \ldots, \vec{z}_{k}, \vec{w}_{1}, \ldots, \vec{w}_{k}$, if $\mathrm{Eq}\left(\vec{x}_{1}, \ldots, \vec{x}_{k}, \vec{z}_{1}, \ldots, \vec{z}_{k}\right)$ and $\operatorname{Eq}\left(\vec{y}_{1}, \ldots, \vec{y}_{k}, \vec{w}_{1}, \ldots, \vec{w}_{k}\right)$ then

$$
\overline{\mathscr{O}_{\vec{z}_{1}} \cap \cdots \cap \mathscr{O}_{\vec{z}_{k}}} \cap \overline{\mathscr{O}_{\vec{w}_{1}} \cap \cdots \cap \mathscr{O}_{\vec{w}_{k}}} \cap E^{*} \neq \varnothing .
$$

It then follows from Claims 4.11 (i) and 4.12 that (Vert, Eq, Edge) semantically embeds $\mathscr{G}$ in $\mathbf{C}$. Hence $\operatorname{SP}\left(\mathbf{A}^{t}\right)$ is hereditarily undecidable.

\section{UNARY CLASSES}

In this section we specialize our results to the case of a locally finite universal class whose type consists of finitely many unary operation symbols. The algebras in such a class are easy to visualize. If $\mathbf{A}$ is a unary algebra and $a, b \in A$ write $a \leq b$ to mean $a \in \operatorname{Sg}_{\mathbf{A}}(b), a \approx b$ to mean $\operatorname{Sg}_{\mathbf{A}}(a)=\operatorname{Sg}_{\mathbf{A}}(b), a<b$ to mean $a \leq b$ but $a \neq b$, and $[a]$ to denote the set $\{x \in A: x \approx a\}$. $\leq$ is a preorder on $A ; \approx$ is its corresponding equivalence relation, and the set $[\mathbf{A}]=\{[a]: a \in A\}$ of $\approx$-classes is a poset in the natural way. Note that the height of this poset is bounded by the maximum size of the 1-generated subalgebras of $\mathbf{A}$; and the subalgebras of $\mathbf{A}$ correspond naturally to the nonempty lower segments of $[\mathbf{A}]$.

Definition 5.1. Let $\mathbf{A}$ be an algebra of finite unary type and let $a, b \in A$.

(1) $D_{a}=\{x \in A: x<a\}$.

(2) $D_{\mathrm{A}}=\bigcup_{a \in A} D_{a}$.

(3) $b$ is a conjugate of $a$ (in A) if $D_{b}=D_{a}$ and there exists an isomorphism $\sigma: \mathbf{S g}_{\mathbf{A}}(a) \cong \mathbf{S g}_{\mathbf{A}}(b)$ satisfying $\sigma(a)=b$ and $\sigma(d)=d$ for all $d \in D_{a}$.

(4) $\operatorname{Conj}_{\mathbf{A}}(a)$ is the set of all conjugates of $a$ in $\mathbf{A}$.

Note that in the previous definition, $D_{a}$ and $D_{\mathbf{A}}$ are subuniverses of $\mathbf{A}$, and the relation "is a conjugate of" is an equivalence relation. The next lemma illustrates the concepts defined above.

Lemma 5.2. Suppose $\mathscr{K}$ is a locally finite universal class of unary algebras of finite type, and $\mathbf{A} \in \mathscr{K}$. If $A_{0}$ is a subuniverse of $\mathbf{A}$ satisfying $D_{\mathbf{A}} \subseteq A_{0}$, then A is locally homogeneous over $A_{0}$.

Proof. Let $D=D_{\mathrm{A}}$. We shall give the proof for the case $A_{0}=D$ (the proof of the general case is essentially the same). Let $L=\{[a]: a \in A \backslash D\}$; thus $L$ is the set of maximal elements of the poset $[\mathbf{A}]$. For $[a],[b] \in L$ let us say that $[a],[b]$ have the same color if $[b] \cap \operatorname{Conj}_{\mathbf{A}}(a) \neq \varnothing$. "Having the same color" is an equivalence relation on $L$. Now suppose $\mathbf{B}_{1}, \mathbf{B}_{2} \leq \mathbf{A}$ with $B_{1} \cap D=B_{2} \cap D$. Isomorphisms from $\mathbf{B}_{1}$ to $\mathbf{B}_{2}$ fixing $B_{1} \cap D$ are particularly easy to describe. Let $L_{i}=\left\{[b] \in L: b \in B_{i}\right\}$ for $i=1,2$, and suppose $\sigma: \mathbf{B}_{1} \cong \mathbf{B}_{2}$ satisfies $\left.\sigma\right|_{B_{1} \cap D}=\operatorname{id}_{B_{1} \cap D}$. Then

1. $\sigma$ induces a bijection $\hat{\sigma}$ from $L_{1}$ to $L_{2}$ by the rule $\hat{\sigma}([b])=[\sigma(b)]$.

2. $\hat{\sigma}$ preserves colors.

3. For each $[b] \in L_{1}$, the restriction $\left.\sigma\right|_{[b]}:[b] \rightarrow[\sigma(b)]$ is a bijection; moreover, $x$ is a conjugate of $\sigma(x)$ for all $x \in[b]$. 
Conversely, if $\tau$ is a color-preserving bijection from $L_{1}$ to $L_{2},\left(b_{\alpha}\right)_{\alpha<\eta}$ is a sequence of elements from $B_{1}$ such that the sequence $\left(\left[b_{\alpha}\right]\right)_{\alpha<\eta}$ names each member of $L_{1}$ exactly once, and $\left(c_{\alpha}\right)_{\alpha<\eta}$ is a sequence from $L_{2}$ such that $c_{\alpha} \in \tau\left(\left[b_{\alpha}\right]\right)$ and $c_{\alpha}$ is a conjugate of $b_{\alpha}$ for all $\alpha<\eta$, then there is a unique isomorphism $\sigma: \mathbf{B}_{1} \cong \mathbf{B}_{2}$ satisfying $\left.\sigma\right|_{B_{1} \cap D}=\operatorname{id}_{B_{1} \cap D}, \hat{\sigma}=\tau$, and $\sigma\left(b_{\alpha}\right)=c_{\alpha}$ for all $\alpha<\eta$.

Now to establish that $\mathbf{A}$ is locally homogeneous over $D$, suppose $\mathbf{C}$ is a finite subalgebra of $\mathbf{A}$ containing $\mathbf{B}_{1}$ and $\mathbf{B}_{2}$, and suppose $\sigma: \mathbf{B}_{1} \cong \mathbf{B}_{2}$ satisfies $\left.\sigma\right|_{B_{1} \cap D}=\left.\mathrm{id}\right|_{B_{1} \cap D}$. Let $L_{1}, L_{2}$ and $\hat{\sigma}$ be as in the previous discussion, let $\left(b_{\alpha}\right)_{\alpha<\eta}$ be a sequence from $B_{1}$ enumerating the $\approx$-classes in $L_{1}$, and define $c_{\alpha}=\sigma\left(b_{\alpha}\right)$ for $\alpha<\eta$. Finally let $L_{3}=\{[b] \in L: b \in C\}$.

Clearly $\hat{\sigma}$ can be extended to a color-preserving bijection $\tau: L_{3} \rightarrow L_{3}$, and the sequences $\left(b_{\alpha}\right)_{\alpha<\eta},\left(c_{\alpha}\right)_{\alpha<\eta}$ can be extended to sequences $\left(b_{\alpha}\right)_{\alpha<\kappa},\left(c_{\alpha}\right)_{\alpha<\kappa}$, both enumerating the $\approx$-classes in $L_{3}$ and such that $c_{\alpha} \in \tau\left(\left[b_{\alpha}\right]\right)$ and $c_{\alpha}$ is a conjugate of $b_{\alpha}$ for all $\alpha<\kappa$. Hence $\sigma$ can be extended to an automorphism $\sigma^{+}$of $\mathbf{C}$ satisfying $\left.\sigma^{+}\right|_{C \cap D}=\operatorname{id}_{C \cap D}$, as desired.

Now we state and prove the principal result of this paper.

Theorem 5.3. Suppose $\mathscr{K}$ is a locally finite universal class of unary algebras of finite type. Then the following are equivalent:

(1) $\mathrm{V}\left(\mathscr{K}^{t}\right)$ is decidable.

(2) $\mathrm{V}\left(\mathscr{K}^{t}\right)$ is not hereditarily undecidable.

(3) $\mathscr{K}$ is almost locally homogeneous.

(4) There exists $N<\omega$ such that for all $\mathbf{A} \in \mathscr{K}$, if $a, c \in A$ with $a<c$, then $\left|\operatorname{Conj}_{\mathbf{A}}(a)\right| \leq N$.

Proof. (1) $\Rightarrow(2)$ is trivial. We shall prove $(4) \Rightarrow(1)$ and (3), and $\neg(4) \Rightarrow$ $\neg(2)$ and $\neg(3)$.

$\neg(4) \Rightarrow \neg(2)$ and $\neg(3)$. Suppose no $N$ exists as in (4). Let $L$ be the type of $\mathscr{K}$. Because there are only finitely many quantifier-free 1-types realized in $\mathscr{K}$, the following predicates are definable in $\mathscr{K}$ by quantifier-free L-formulas:

1. $x \leq y$ (and thus $x \approx y$ and $x<y$ );

2. $x$ and $y$ are conjugates.

Hence the assumption that $N$ fails to exist, combined with a compactness argument, produces a countable algebra $\mathbf{B} \in \mathscr{K}$ and elements $a, b \in B$ such that $\operatorname{Conj}_{\mathbf{B}}(a)$ is infinite and $a<b$. Let $M_{0}=\operatorname{Conj}_{\mathbf{B}}(a)$. As the poset [B] has finite height, we may assume that $a$ and $b$ have been chosen so that if $m \in M_{0}$ and $m \leq b$, then $[m] \prec[b]$. Define

$$
\begin{aligned}
M & =\left\{x \in B: x \approx m \text { for some } m \in M_{0}\right\}, \quad A=\operatorname{Sg}_{\mathbf{B}}(M \cup\{b\}), \\
P & =D_{a}, \quad S=\operatorname{Sg}_{\mathbf{B}}(M)=M \cup P, \quad T=\{m \in M: m<b\} .
\end{aligned}
$$

See Figure 1 for a schematic representation of the poset $[\mathbf{A}]$.

Note that $\operatorname{Conj}_{\mathbf{A}}(a)=\operatorname{Conj}_{\mathbf{S}}(a)=\operatorname{Conj}_{\mathbf{B}}(a)=M_{0}$; that $T$ and $P$ are finite sets (they are contained in a 1-generated subalgebra of $\mathbf{A}$ ) while $M$ is infinite; that $D_{\mathrm{S}}=P$, so $\mathbf{S}$ is homogeneous over $P$ by Lemma 5.2 and Claim 2.2(ii); and that (using the terminology of the proof of Lemma 5.2) the $\approx$-classes in $M$ have the same color.

Again because the number of quantifier-free 1-types realized in $\mathscr{K}$ is finite, there is a quantifier-free L-formula $\mu_{0}(x)$ with parameters from $P$ such that 


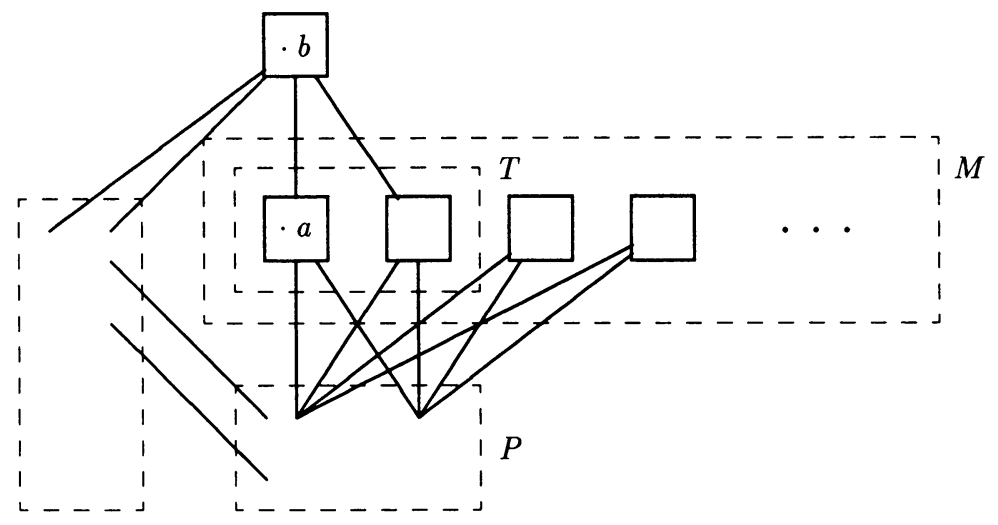

FIGURE 1. The poset [A]

$\mu_{0}^{\mathbf{A}}=M_{0}$. Let $\mu(x), \tau(x)$, and $\psi(x)$ be the formulas

$$
\begin{aligned}
\mu(x) & : \exists y\left[\mu_{0}(y) \& x \approx y\right], \\
\tau(x) & : \mu(x) \& \exists z[x<z], \\
\psi(z) & : \exists x[\mu(x) \& x<z] .
\end{aligned}
$$

Clearly $\left.\mu^{\mathbf{A}}\right|_{S}=\mu^{\mathbf{A}}=M,\left.\tau^{\mathbf{A}}\right|_{S}=T$, and $b \in \psi^{\mathbf{A}}$ while $\left.\psi^{\mathbf{A}}\right|_{S}=\varnothing$.

Suppose $m \in M_{0}$; as $a$ and $m$ are conjugates, there is an isomorphism $\alpha: \mathbf{S g}_{\mathbf{S}}(a) \cong \mathbf{S g}_{\mathbf{S}}(m)$ satisfying $\left.\alpha\right|_{P}=\operatorname{id}_{P}$ and $\alpha(a)=m$. As $\mathbf{S}$ is homogeneous over $P, \alpha$ extends to an isomorphism $\sigma$ of $\mathbf{S}$ (which fixes $P$ ). Clearly $\sigma([a])=[m]$ and $\sigma(M)=M$; hence

$$
M=\bigcup\left\{\sigma(T): \sigma \in \text { Aut } \mathbf{S}:\left.\sigma\right|_{P}=\operatorname{id}_{P} \text { and } \sigma(M)=M\right\} .
$$

It follows from the above remarks that if the language $L$ is expanded to include a family of new constant symbols indexed by $P$, and if $\mathbf{A}^{*}$ and $\mathbf{S}^{*}$ are the natural expansions of $\mathbf{A}$ and $\mathbf{S}$ to this larger language, then $\mathbf{A}^{*}, \mathbf{S}^{*}, \mu, \tau$, and $\psi$ satisfy the hypotheses of Lemma 4.1 ( $\equiv$ is equality). So a hereditarily undecidable class of graphs semantically embeds into $\operatorname{SP}\left(\left(\mathbf{A}^{*}\right)^{t}\right)$, from which it follows that $\mathrm{V}\left(\mathscr{K}^{t}\right)$ is hereditarily undecidable. This proves $\neg(2)$.

To prove $\neg(3)$, suppose $\mathscr{K}$ were almost locally homogeneous and $\mathscr{K}_{0}$ witnessed this fact. Let $S_{0}$ be a maximal $\mathscr{K}_{0}$-subuniverse of $\mathbf{S}$; extend $S_{0}$ to a maximal $\mathscr{H}_{0}$-subuniverse $A_{0}$ of $\mathbf{A}$. Then by hypothesis and Claim 2.2(ii), A is locally homogeneous over $A_{0}, A_{0} \cap S=S_{0}$, and $S_{0}$ is finite.

Fix $m \in M_{0} \backslash T$. As $a$ and $m$ are conjugates, there is an isomorphism $\alpha: \mathbf{S g}_{\mathbf{A}}(a) \cong \mathbf{S g}_{\mathbf{A}}(m)$ satisfying $\left.\alpha\right|_{P}=\operatorname{id}_{P}$ and $\alpha(a)=m$. But clearly $\alpha$ cannot be extended to an automorphism of $\mathbf{A}$, by the choice of $m$. Because A is homogeneous over $A_{0}$, it must be the case that either or

1. $\operatorname{Sg}_{\mathbf{A}}(a) \cap S_{0} \neq \operatorname{Sg}_{\mathbf{A}}(m) \cap S_{0}$,

2. $\operatorname{Sg}_{\mathbf{A}}(a) \cap S_{0}=\operatorname{Sg}_{\mathbf{A}}(m) \cap S_{0}=E$ but $\left.\alpha\right|_{E} \neq \mathrm{id}_{E}$.

Note that if $\operatorname{Sg}_{\mathrm{A}}(a) \cap S_{0}=\operatorname{Sg}_{\mathrm{A}}(m) \cap S_{0}=E$ then necessarily $E \subseteq P$; so the fact that $\left.\alpha\right|_{P}=\operatorname{id}_{P}$ implies that condition (2) cannot hold. Thus $\operatorname{Sg}_{\mathrm{A}}(a) \cap S_{0} \neq$ $\mathrm{Sg}_{\mathrm{A}}(m) \cap S_{0}$, and hence $[a] \subseteq S_{0}$ or $[m] \subseteq S_{0}$. If $a \notin S_{0}$ then this argument 
shows that $m \in S_{0}$ for all $m \in M_{0} \backslash T$, which is impossible as $S_{0}$ is finite. Thus $a \in S_{0}$.

It was shown in the proof of $\neg(2)$ that for any $m \in M_{0}$ there exists $\sigma \in$ Aut $\mathbf{S}$ satisfying $\sigma(a)=m$. Clearly $\sigma^{-1}\left(S_{0}\right)$, like $S_{0}$, is a maximal $\mathscr{K}_{0}$-subuniverse of $\mathbf{S}$. Then the above argument applied to $\sigma^{-1}\left(S_{0}\right)$ shows that $a \in \sigma^{-1}\left(S_{0}\right)$, or equivalent, $m \in S_{0}$. Hence $M_{0} \subseteq S_{0}$, again contradicting the finiteness of $S_{0}$. So $\mathscr{K}$ is not almost locally homogeneous.

(4) $\Rightarrow(1)$ and (3). Suppose that $N$ exists as in (4). Let $M$ be the maximum cardinality of the 1 -generated members of $\mathscr{K}$, and let $\mathscr{H}$ be the class of all L-algebras $A$ such that:

1. If $a, c \in A$ with $a<c$, then $\left|\operatorname{Conj}_{\mathbf{A}}(a)\right| \leq N$.

2. Every 1-generated subalgebra of $\mathbf{A}$ has cardinality at most $M$.

Note that $\mathscr{H}$ is a finitely axiomatizable locally finite universal class and $\mathscr{K} \subseteq$ $\mathscr{H}$. Let $\mathscr{H}^{\prime}$ be the class of all $\mathbf{A} \in \mathscr{H}$ in which $\left|\operatorname{Conj}_{\mathbf{A}}(a)\right| \leq N$ for all $a \in A$. Note that if $\mathbf{A} \in \mathscr{H}$ and $D=D_{\mathbf{A}} \neq \varnothing$, then $\mathbf{D} \in \mathscr{H}^{\prime}$.

Claim 5.4. $\mathscr{H}^{\prime}=\mathrm{I}\left(\mathscr{H}_{0}\right)$ for some finite set $\mathscr{H}_{0}$ of finite algebras.

Proof. It will suffice to show that for every $i \leq M$ there is a positive integer $n_{i}$ with the property that $|A| \leq n_{i}$ for all $\mathbf{A} \in \mathscr{H}^{\prime}$ such that height $([\mathbf{A}])=i$. The proof is by induction on $i$. As in the discussion preceding Definition 2.5, let $T_{1}=\left\{t_{0}, \ldots, t_{r-1}\right\}$ be a finite set of unary terms with the property that for all $\mathbf{A} \in \mathscr{H}$ and $a \in A, \operatorname{Sg}_{\mathbf{A}}(a)=\left\{t_{i}^{\mathbf{A}}(a): i<r\right\}$. Also define $n_{-1}=0$. Now suppose $\mathbf{A} \in \mathscr{H}^{\prime}$ with height $([\mathbf{A}])=i \geq 0$, and let $D=D_{\mathbf{A}}$. Then $|D| \leq n_{i-1}$. Let $L=\{[a]: a \in A \backslash D\}$ be as in the proof of Lemma 5.2, and for $[a] \in L$ let us call the set $\{[b] \in L:[b]$ has the same color as $[a]\}$ a color-class of $\mathbf{A}$.

Assume $\infty \notin D$ and for each $b \in A \backslash D$ define a map $\delta_{b}: T_{1} \rightarrow D \cup\{\infty\}$ by

$$
\delta_{b}\left(t_{i}\right)= \begin{cases}\infty & \text { if } t_{i}^{\mathbf{A}}(b) \approx b, \\ t_{i}^{\mathbf{A}}(b) & \text { otherwise. }\end{cases}
$$

It is easy to see that if $b, c \in A \backslash D$ and $b, c$ have the same quantifier-free 1type, then $b, c$ are conjugates iff $\delta_{b}=\delta_{c}$ (in which case [b], [c] have the same color). So the number of distinct color-classes of $\mathbf{A}$ is at most $s \cdot\left|(D \cup\{\infty\})^{T_{1}}\right| \leq$ $s\left(1+n_{i-1}\right)^{r}$, where $s$ is the number of quantifier-free 1-types realized in $\mathscr{H}$ (and $\left.r=\left|T_{1}\right|\right)$. On the other hand, each color-class of $\mathbf{A}$ has at most $N$ elements by definition of $\mathscr{H}^{\prime}$. Thus $|A \backslash D| \leq M \cdot|L| \leq M N s\left(1+n_{i-1}\right)^{r}$, and so the integer $n_{i}=n_{i-1}+M N s\left(1+n_{i-1}\right)^{r}$ satisfies the desired condition.

Claim 5.5. $\mathscr{H}$ is almost locally homogeneous.

Proof. We shall verify the conditions of Definition 3.2 using the set $\mathscr{H}_{0}$ mentioned in the previous claim. The first two conditions are easily proved, so we concentrate on the third. Let $\mathbf{A} \in \mathscr{H}$ and suppose $A_{0}$ is a maximal $\mathscr{H}_{0}$ subuniverse of $\mathbf{A}$. Put $D=D_{\mathrm{A}}$ and $B=A_{0} \cup D$. It is easy to check that $\mathbf{B} \in \mathscr{H}^{\prime}$ if $B \neq \varnothing$, so $B=A_{0}$ by maximality. Then by Lemma $5.2, \mathbf{A}$ is locally homogeneous over $A_{0}$, verifying the third condition.

Claim 5.6. Every universal subclass of $\mathscr{H}$ is finitely axiomatizable.

Proof. First note that since $\mathrm{L}$ (the type of $\mathscr{H}$ ) is finite, it follows that for every $\mathbf{A} \in \mathscr{H}_{\text {fin }}$ there is an L-sentence $\phi_{\mathbf{A}}$ with the property that for any universal class $\mathscr{K}$ of type $\mathrm{L}, \mathscr{K} \vDash \phi_{\mathbf{A}}$ iff $\mathbf{A} \notin \mathscr{K}$. Also let $P$ be a complete set of 
representatives of the isomorphism classes of $\mathscr{H}_{\text {fin }}$, and let $\mathbf{P}=\langle P, \hookrightarrow\rangle$ be the corresponding poset under embeddability.

Suppose $\mathscr{K}$ is a universal subclass of $\mathscr{H}$; then $P \cap \mathscr{K}$ is a lower segment of $\mathbf{P}$. As $\mathbf{P}$ satisfies the descending chain condition, the complementary upper segment $P \backslash \mathscr{K}$ is generated by its antichain $\mathscr{C}$ of minimal elements. As every locally finite algebra is an ultraproduct of its finite subalgebras, $\mathscr{K}$ is axiomatized relative to $\mathscr{H}$ by the set $\left\{\phi_{\mathbf{A}}: \mathbf{A} \in \mathscr{C}\right\}$. Thus to prove the claim it is enough to show that $\mathbf{P}$ has no infinite antichains.

For each $\mathbf{B} \in \mathscr{H}_{0}$ let $\mathscr{H}_{\mathbf{B}}=\left\{\mathbf{A} \in \mathscr{H}_{\text {fin }}: \mathbf{D}_{\mathbf{A}}=\mathbf{B}\right\}$. Also let $\mathscr{H}_{\varnothing}=\{\mathbf{A} \in$ $\left.\mathscr{H}_{\text {fin }}: D_{\mathrm{A}}=\varnothing\right\}$. In what follows, we shall refer to B-embeddings between members of $\mathscr{H}_{\mathbf{B}}$, and to quantifier-free $\mathbf{B}$-1-types realized in $\mathscr{H}_{\mathbf{B}}$. These notions were defined in $\S 2$.

Fix $\mathbf{B} \in \mathscr{H}_{0} \cup\{\varnothing\}$, and let $p_{0}, \ldots, p_{s-1}$ enumerate the quantifier-free $\mathbf{B}-1$ types realized in $\mathscr{H}_{\mathbf{B}}$. For each $\mathbf{A} \in \mathscr{H}_{\mathbf{B}}$ let $L=\left\{[a]: a \in A \backslash D_{\mathbf{A}}\right\}$ and define $\alpha_{\mathbf{A}}:\{0, \ldots, s-1\} \rightarrow \omega$ by

$\alpha_{\mathbf{A}}(i)=\mid\left\{[a] \in L\right.$ : there exists $b \in[a]$ whose quantifier-free B-1-type is $\left.p_{i}\right\}$.

The key is to observe that if $\mathbf{A}, \mathbf{A}^{\prime} \in \mathscr{H}_{\mathbf{B}}$ then $\mathbf{A} \stackrel{\mathbf{B}}{\hookrightarrow} \mathbf{A}^{\prime}$ iff $\alpha_{\mathbf{A}} \leq \alpha_{\mathbf{A}^{\prime}}$ in the product ordering of $\omega^{s}$.

Now suppose that $\left\{\mathbf{A}_{0}, \mathbf{A}_{1}, \ldots\right\}$ is an infinite antichain in $\mathbf{P}$. We may assume that each $\mathbf{A}_{i}$ belongs to $\mathscr{H}_{\mathbf{B}}$ for some $\mathbf{B} \in \mathscr{H}_{0} \cup\{\varnothing\}$; and as $\mathscr{H}_{0} \cup\{\varnothing\}$ is finite we can further assume that one of the $\mathscr{H}_{\mathbf{B}}$ s contains all of the $\mathbf{A}_{i}$ s. Now for any positive integer $s, \omega^{s}$ has no infinite antichains; so there must exist $i \neq j$ for which $\alpha_{\mathbf{A}_{i}} \leq \alpha_{\mathbf{A}_{j}}$. But then $\mathbf{A}_{i} \stackrel{\mathbf{B}}{\hookrightarrow} \mathbf{A}_{j}$ by the above observation, and so certainly $\mathbf{A}_{i} \hookrightarrow \mathbf{A}_{j}$, which contradicts the supposition that the $\mathbf{A}_{i}$ s form an antichain. This proves the claim.

Now we can finish the proof of (1) and (3). $\mathscr{K}$ is finitely axiomatizable by Claim 5.6; hence $\operatorname{Th}_{\forall}(\mathscr{K})$ is decidable. As the property of being almost locally homogeneous is inherited by universal subclasses, $\mathscr{K}$ is almost locally homogeneous by Claim 5.5. Thus $\vee\left(\mathscr{K}^{t}\right)$ is decidable by Theorem 3.3. This finishes the proof of Theorem 5.3.

\section{CONClusion}

The methods developed in this paper to study the decidability of $\mathrm{V}\left(\mathscr{K}^{t}\right)$ have applications beyond the case when $\mathscr{K}$ is of unary type. For example, $M$. Valeriote and the author [20] have used the semantic embedding of $\S 4$ to show that if ${ }_{R} \mathscr{M}$ is the variety of all unitary left $R$-modules for some finite ring $R$ with unit, then $\vee\left({ }_{R} \mathscr{M}^{t}\right)$ is decidable iff $R$ is semisimple. This finishes the classification, nearly completed in [4], of those locally finite varieties $\mathscr{V}$ of finite type for which $\mathrm{V}\left(\mathscr{V}^{t}\right)$ is decidable.

A second application is to the study of the decidability of the finite members of $\mathrm{V}\left(\mathscr{K}^{t}\right)$, where $\mathscr{K}$ is a locally finite universal class of finite type. By the theorem of Bulman-Fleming and Werner, $\mathrm{V}\left(\mathscr{K}^{t}\right)_{\text {fin }}$ consists of all algebras isomorphic to finite direct products of members of $\mathscr{K}_{\text {fin }}^{t}$, plus 1-element algebras. Hence an easy argument using the original theorem of Feferman and Vaught [11] proves that $\mathrm{V}\left(\mathscr{K}^{t}\right)_{\text {fin }}$ is decidable iff $\mathscr{K}_{\text {fin }}^{t}$ is decidable (iff $\mathscr{K}_{\text {fin }}$ is decidable). 
Our methods in $\S \S 2$ and 3 can be used to show that if $\mathscr{K}$ is almost locally homogeneous, then $\operatorname{Th}(\mathscr{K})=\operatorname{Th}\left(\mathscr{K}_{\text {fin }}\right)$, and both are decidable if $\operatorname{Th}_{\forall}(\mathscr{K})$ is decidable. It follows from Theorem 5.3 that if $\mathscr{K}$ is of unary type, then the decidability of $\mathrm{V}\left(\mathscr{K}^{t}\right)$ implies the decidability of the finite members of $\mathrm{V}\left(\mathscr{K}^{t}\right)$.

Joohee Jeong at the University of California, Berkeley, recently discovered [12] an example of a universal class $\mathscr{K}$ of unary algebras of finite type with the property that $\mathrm{V}\left(\mathscr{K}^{t}\right)$ is decidable while $\mathrm{V}\left(\mathscr{K}^{t}\right)_{\text {fin }}$ is not. By the previous remarks, $\mathscr{K}$ cannot be locally finite. It would be interesting to know whether there exists a locally finite universal class of finite (but not unary) type with this same property.

Finally, we mention that we know of no locally finite universal class $\mathscr{K}$ of finite type for which $\mathrm{Th}_{\forall}(\mathscr{K})$ is decidable and which is a counterexample to the converse of Theorem 3.3. The discovery of such a counterexample (or a proof that none exists) would greatly increase our understanding of decidable discriminator varieties.

Added in Proof. A counterexample to the converse of Theorem 3.3 may be found in [23].

\section{REFERENCES}

1. S. Bulman-Fleming and $\mathrm{H}$. Werner, Equational compactness in quasiprimal varieties, Algebra Universalis 7 (1977), 33-46.

2. S. Burris, Iterated discriminator varieties have undecidable theories, Algebra Universalis 21 (1985), 54-61.

3. S. Burris and R. McKenzie, Decidability and Boolean representations, Mem. Amer. Math. Soc., vol. 246, 1981.

4. S. Burris, R. McKenzie, and M. Valeriote, Decidable discriminator varieties from unary varieties, J. Symbolic Logic 56 (1991), 1355-1368.

5. S. Burris and H. P. Sankappanavar, A course in universal algebra, Springer-Verlag, New York, 1981.

6. S. Burris and $\mathrm{H}$. Werner, Sheaf constructions and their elementary properties, Trans. Amer. Math. Soc. 248 (1979), 269-309.

7. S. Comer, Elementary properties of structures of sections, Bol. Soc. Mat. Mexicana 19 (1974), 78-85.

8. __ Monadic algebras with finite degree, Algebra Universalis 5 (1975), 315-327.

9. Yu. Ershov, Decidability of the elementary theory of relatively complemented lattices and of the theory of filters, Algebra i Logika 3 (1964), 17-38.

10. __ Elementary theory of Post varieties, Algebra i Logika 6 (1967), 7-15.

11. S. Feferman and R. L. Vaught, The first order properties of products of algebraic systems, Fund. Math. 47 (1959), 57-103.

12. J. Jeong, A decidable variety that is not finitely decidable, J. Symbolic Logic (to appear).

13. K. Keimel and $\mathrm{H}$. Werner, Stone duality for varieties generated by quasi primal algebras, Mem. Amer. Math. Soc., no. 148, 1974, pp. 59-85.

14. R. McKenzie, G. McNulty, and W. Taylor, Algebras, lattices, varieties, vol. I, Wadsworth \& Brooks/Cole, Monterey, Calif., 1987.

15. R. McKenzie and M. Valeriote, The structure of decidable locally finite varieties, Birkhäuser, Boston, Mass., 1989.

16. M. O. Rabin, Decidability of second order theories and automata on infinite trees, Trans. Amer. Math. Soc. 141 (1969), 1-35.

17. M. Rubin, The theory of Boolean algebras with a distinguished subalgebra is undecidable, Ann. Sci. Univ. Clermont-Ferrand II Math., no. 60 (1976), 129-134. 
18. A. Tarski, Arithmetical classes and types of Boolean algebras, Bull. Amer. Math. Soc. 55 (1949), 64.

19. M. Valeriote, On decidable locally finite varieties, Ph.D. thesis, Univ. of California, Berkeley, 1986.

20. M. Valeriote and R. Willard, Discriminating varieties, Algebra Universalis (to appear).

21. V. Weispfennig, A note on aleph-0-categorical model companions, Arch. Math. Logic 19 (1978), 23-29.

22. $\mathrm{H}$. Werner, Varieties generated by quasiprimal algebras have decidable theories, Contributions to Universal Algebra (B. Csákány and J. Schmidt, eds.), Math. Soc. János Bolyai, vol. 17, North-Holland, 1977, pp. 555-575.

23. R.Willard, Decidable discriminator varieties with lattice stalks, Algebra Universalis (to appear).

Department of Mathematics, Carnegie Mellon University, Pittsburgh, Pennsylvania 15213-3890

Current address: Department of Pure Mathematics, University of Waterloo, Waterloo, Ontario, Canada N2L 3G1

E-mail address: rdwillard@dragon.waterloo.edu 